\title{
More is Less: Perfectly Secure Oblivious Algorithms in the Multi-Server Setting
}

\author{
T-H. Hubert Chan ${ }^{1}$, Jonathan $\mathrm{Katz}^{2}$, Kartik Nayak ${ }^{2,3}$, Antigoni \\ Polychroniadou ${ }^{4}$, and Elaine $\mathrm{Shi}^{5}$ \\ 1 The University of Hong Kong, hubert@cs.hku.hk \\ 2 University of Maryland, College Park, jkatz@cs.umd.edu \\ 3 VMware Research, nkartik@vmware.com \\ ${ }^{4}$ Cornell Tech, antigoni@cornell.edu \\ 5 Cornell University, runting@gmail.com
}

\begin{abstract}
The problem of Oblivious RAM (ORAM) has traditionally been studied in a single-server setting, but more recently the multi-server setting has also been considered. Yet it is still unclear whether the multiserver setting has any inherent advantages, e.g., whether the multi-server setting can be used to achieve stronger security goals or provably better efficiency than is possible in the single-server case.

In this work, we construct a perfectly secure 3-server ORAM scheme that outperforms the best known single-server scheme by a logarithmic factor. In the process we also show, for the first time, that there exist specific algorithms for which multiple servers can overcome known lower bounds in the single-server setting.
\end{abstract}

Keywords: Oblivious RAM, perfect security

\section{Introduction}

Oblivious RAM (ORAM) protocols [11] allow a client to outsource storage of its data such that the client can continue to read/write its data while hiding both the data itself as well as the client's access pattern. ORAM was historically considering in a single-server setting, but has recently been considered in a multi-server setting [1, 15, 16, 18, 20, 24] where the client can store its data on multiple, non-colluding servers. Current constructions of multi-server ORAM are more efficient than known protocols in the single-server setting; in particular, the best known protocols in the latter setting (when server-side computation is not allowed) require bandwidth $O\left(\log ^{2} N / \log \log N\right)$ [3, 6, 14, 17, for storing an array of length $N$, whereas multi-server ORAM schemes achieving logarithmic bandwidth6 are known [20].

\footnotetext{
${ }^{6}$ Although Lu and Ostrovsky 20] describe their multi-server scheme using serverside computation, it is not difficult to see that it can be replaced with client-side computation instead.
} 
Nevertheless, there remain several unanswered questions about the multiserver setting. First, all work thus far in the multi-server setting achieves either computational or statistical security, but not perfect security where correctness is required to hold with probability 1 and security must hold even against computationally unbounded attackers. Second, although (as noted above) we have examples of multi-server schemes that beat existing single-server constructions, it is unclear whether this reflects a limitation of our current knowledge or whether there are inherent advantages to the multi-server setting.

We address the above questions in this work. (Unless otherwise noted, our results hold for arbitrary block size $B$ as long as it is large enough to store an address, i.e., $B=\Omega(\log N)$.) First, we construct perfectly secure, multi-server ORAM scheme that improves upon the overhead of the best known construction in the single-server setting. Specifically, we show:

Theorem 1. There exists a 3-server ORAM scheme that is perfectly secure for any single semi-honest corruption, and achieves $O\left(\log ^{2} N\right)$ bandwidth per logical memory access on an array of length $N$. Further, our scheme does not rely on server-side computation.

As a point of comparison, the best known single-server, perfectly secure ORAM schemes require $O\left(\log ^{3} N\right)$ bandwidth [5, 8. While Theorem 11 holds for any block size $B=\Omega(\log N)$, we show that for block sizes $B=\Omega\left(\log ^{2} N\right)$ our scheme achieves bandwidth as small as $O(\log N)$.

As part of our construction, we introduce new building blocks that are of independent theoretical interest. Specifically, we show:

Theorem 2. There exists a 3-server protocol for stable compaction that is perfectly secure for any single semi-honest corruption, and achieves $O(N)$ bandwidth to compact an array of length $N$ (that is secret shared among the servers). The same result holds for merging two sorted arrays of length $N$.

In the single-server setting, Lin, Shi, and Xie [19 recently proved a lower bound showing that any oblivious algorithm for stable compaction or merging in the balls-and-bins model must incur at least $\Omega(N \log N)$ bandwidth. The balls-and-bins model characterizes a wide class of natural algorithms where each element is treated as an atomic "ball" with a numeric label; the algorithm may perform arbitrary boolean computation on the labels, but is only allowed to move the balls around and not compute on their values. Our scheme works in the balls-and-bins model, and thus shows for the first time that the multiserver setting can enable overcoming known lower bounds in the single-server setting for oblivious algorithms. Furthermore, for stable compaction and merging no previous multi-server scheme was known that is asymptotically faster than existing single-server algorithms, even in the weaker setting of computational security. We note finally that our protocols are asymptotically optimal since clearly any correct algorithm has to read the entire array. 


\subsection{Technical Roadmap}

Oblivious sorting is an essential building block in hierarchical ORAM schemes. At a high level, the key idea is to replace oblivious sorting, which $\operatorname{costs} O(n \log n)$ time on an array of length $n$, with cheaper, linear-time operations. Indeed, this was also the idea of Lu and Ostrovsky [20, but they apply it to a computationally secure hierarchical ORAM. Earlier single-server ORAM schemes are built from logarithmically many cuckoo hash tables of doubling size. Every time a memory request has been served, one needs to merge multiple stale cuckoo hash tables into a newly constructed cuckoo hash table - this was previously accomplished by oblivious sorting [3, 14, 17. Lu and Ostrovsky show how to avoid cuckoo hashing, by having one permutation server permute the data in linear time, and by having a separate storage server, that is unaware of the permutation, construct a cuckoo hash table from the permuted array in linear time (with the client's help). Unfortunately, Lu and Ostrovsky's technique fails for the perfect security context due to its intimate reliance on pseudorandom functions (PRFs) and cuckoo hashing - the former introduces computational assumptions and the latter leads to statistical failures (albeit with negligible probability).

We are, however, inspired by Lu and Ostrovsky's permutation-storage-separation paradigm (and a similar approach that was described independently by Stefanov and Shi 24]). The key concept here is to have one permutation-server that permutes the data; and have operations and accesses be performed by a separate storage server that is unaware of the permutation applied. One natural question is whether we can apply this technique to directly construct a linear-time multiserver oblivious sorting algorithm - unfortunately we are not aware of any way to achieve this. Chan et al. 44 and Tople et al. 26] show that assuming the data is already randomly permuted (and the permutation hidden), one can simply apply any comparison-based sorting algorithm and it would retain obliviousness. Unfortunately, it is well-known that comparison-based sorting must incur $\Omega(n \log n)$ time, and this observation does not extend to non-comparison-based sorting techniques since in general RAM computations on numeric keys can leak information through access patterns.

New techniques at a glance. We propose two novel techniques that allow us to achieve the stated results, both of which rely on the permutation-storageseparation paradigm:

- First, we observe that with multiple servers, we can adapt the single-server perfect ORAM scheme by Chan et al. 5 into a new variant such that reshuffling operations which was realized with oblivious sorting in Chan et al. [5] can now be expressed entirely with merging and stable compaction operations without oblivious sorting.

- Despite the known lower bounds in the single-server setting [19], we show that with multiple servers, we can indeed achieve linear-time oblivious merging and oblivious stable compaction. As many have observed earlier [3, 4, 6, 13, merging and compaction are also important building blocks in the design of 
many oblivious algorithms - we thus believe that our new building blocks are of independent interest.

Stable Compaction and Merging. We first explain the intuition behind our stable compaction algorithm. For simplicity, for the time being we will consider only 2 servers and assume perfectly secure encryption for free (this assumption can later be removed with secret sharing and by introducing one additional server). Imagine that we start out with an array of length $N$ that is encrypted and resides on one server. The elements in the array are either real or dummy, and we would like to move all dummy elements to the end of the array while preserving the order of the real elements as they appear in the original array. For security, we would like that any single server's view in the protocol leaks no information about the array's contents.

Strawman scheme. An extremely simple strawman scheme is the following: the client makes a scan of the input array on one server; whenever it encounters a real element, it re-encrypts it and writes it to the other server by appending it to the end of the output array (initially the output array is empty). When the entire input array has been consumed, the client pads the output array with an appropriate number of (encrypted) dummy elements.

At first sight, this algorithms seems to preserve security: each server basically observes a linear scan of either the input or the output array; and the perfectly secure encryption hides array contents. However, upon careful examination, the second server can observe the time steps in which a write happened to the output array - and this leaks which elements are real in the original array. Correspondingly, in our formal modeling later (Section 2), each server cannot only observe each message sent and received by itself, but also the time steps in which these events occurred.

A second try. For simplicity we will describe our approach with server computation and server-to-server communication - but it is not hard to modify the scheme such that servers are completely passive. Roughly speaking, the idea is for the first server (called the permutation server) to randomly permute all elements and store a permuted array on the second server (called the storage server), such that the permutation is hidden from the storage server. Moreover, in this permuted array, we would like the elements to be tagged with pointers to form two linked lists: a real linked list and a dummy linked list. In both linked lists, the ordering of elements respects that of the original array. If such a permuted array encoding two linked lists can be constructed, the client can simply traverse the real linked list first from the storage server, and then traverse the dummy linked list — writing down each element it encounters on the first server (we always assume re-encryption upon writes). Since the storage server does not know the random permutation and since every element is accessed exactly once, it observes completely random access patterns; and thus it cannot gain any secret information. 
The challenge remains as to how to tag each element with the position of the next element in the permuted array. This can be achieved in the following manner: the permutation server first creates a random permutation in linear time (e.g., by employing Fisher-Yates [10]), such that each element in the input array is now tagged with where it wants to be in the permuted array (henceforth called the position label). Now, the client makes a reverse scan of this input array. During this process, it remembers the position labels of the last real element seen and of the last dummy element seen so far - this takes $O(1)$ client-side storage. Whenever a real element is encountered, the client tags it with the position label of the last real seen. Similarly, whenever a dummy is encountered, the client tags it with the position label of the last dummy seen. Now, the permutation server can permute the array based on the predetermined permutation (which can also be done in linear time). At this moment, it sends the permuted, re-encrypted array to the storage server and the linked list can now be traversed from the storage server to read real elements followed by dummy elements.

It is not difficult to see that assuming that the encryption scheme is perfectly secure and every write involves re-encrypting the data, then the above scheme achieves perfect security against any single semi-honest corrupt server, and completes in linear time. Later we will replace the perfectly secure encryption with secret sharing and this requires the introduction of one additional server.

Extending the idea for merging. We can extend the above idea to allow lineartime oblivious merging of two sorted arrays. The idea is to prepare both arrays such that they are in permuted form on the storage server and in a linked list format; and now the client can traverse the two linked lists on the storage server, merging them in the process. In each step of the merging, only one array is being consumed - since the storage server does not know the permutation, it sees random accesses and cannot tell which array is being consumed.

3-Server Perfectly Secure ORAM. We now explain the techniques for constructing a 3-server perfectly secure ORAM. A client, with $O(1)$ blocks of local cache, stores $N$ blocks of data (secret-shared) on the 3 servers, one of which might be semi-honest corrupt. In every iteration, the client receives a memory request of the form (read, addr) or (write, addr, data), and it completes this request by interacting with the servers. We would like to achieve $O\left(\log ^{2} N\right)$ amortized bandwidth blowup per logical memory request.

We start out from a state-of-the-art single-server perfectly-secure scheme by Chan et al. [5] that achieves $O\left(\log ^{3} N\right)$ amortized bandwidth blowup per memory request. Their scheme follows the hierarchical ORAM paradigm [11,12 and meanwhile relies on a standard recursion technique most commonly adopted by tree-based ORAMs [23. In their construction, there are logarithmically many hierarchical ORAMs (also called position-based ORAMs), where the ORAM at depth $d$ (called the parent depth) stores position labels for the ORAM at depth $d+1$ (called the child depth); and finally, the ORAM at the maximum depth $D=O(\log N)$ stores the real data blocks. 
As it turns out, the most intricate part of Chan et al. [5]'s scheme is the information passing between an ORAM at depth $d$ and its parent ORAM at depth $d-1$. As Chan et al. describe it, all the logarithmically many ORAMs must perform coordinated reshuffles upon every memory request: during a reshuffle, the ORAM at depth $d$ must pass information back to the parent depth $d-1$. More specifically, the depth- $d$ ORAM is aware of the updated position labels for blocks that have been recently visited, and this must be passed back to the parent depth to be recorded there.

More abstractly and somewhat imprecisely, here is a critical building block in Chan et al. [5]: suppose that the parent and the child each has an array of logical addresses and a position label for each address. It is guaranteed by the ORAM construction that all addresses the child has must appear in the parent's array. Moreover, if some address appears in both the parent and child, then the child's version is fresher. Now, we would like to combine the information held by the parent and the child, retaining the freshest copy of position label for every address. Chan et al. then relied on oblivious sorting to achieve this goal: if some address is held by both the parent and child, they will appear adjacent to each other in the sorted array; and thus in a single linear scan one can easily cross out all stale copies.

To save a logarithmic factor, we must solve the above problem using only merging and compaction and not sorting. Notice that if both the parent's and the child's arrays are already sorted according to the addresses, then the aforementioned information propagation from child to parent can be accomplished through merging rather than sorting (in the full scheme we would also need stable compaction to remove dummy blocks in a timely fashion to avoid blowup of array sizes over time). But how can we make sure that these arrays are sorted in the first place without oblivious sorting? In particular, these arrays actually correspond to levels in a hierarchical ORAM in Chan et al. 55's scheme, and all blocks in a level must appear in randomly permuted order to allow safe (onetime) accesses - this seems to contradict our desire for sortedness. Fortunately, here we can rely again on the permutation-storage-separation paradigm — for simplicity again we describe our approach for 2 servers assuming perfectly secure (re-)encryption upon every write. The idea is the following: although the storage server is holding each array (i.e., level) in a randomly permuted order, the permutation server will remember an inverse permutation such that when this permutation is applied to the storage server's copy, sortedness is restored. Thus whenever shuffling is needed, the permutation server would first apply the inverse permutation to the storage server's copy to restore sortedness, and then we could rely on merging (and compaction) to propagate information between adjacent depths rather than sorting.

\subsection{Related Work}

The notion of Oblivious RAM (ORAM) was introduced by the seminal work of Goldreich and Ostrovsky around three decades ago [11,12. Their construction used a hierarchy of buffers of exponentially increasing size, which was later known 
as the hierarchical ORAM framework. Their construction achieved an amortized bandwidth blowup of $O\left(\log ^{3} N\right)$ and was secure against a computationally bounded adversary. Subsequently, several works have improved the bandwidth blowup from $O\left(\log ^{3} N\right)$ to $O\left(\log ^{2} N / \log \log N\right)$ [3]614]17] under the same adversarial model. Ajtai 2 was the first to consider the notion of a statistically secure oblivious RAM that achieves $O\left(\log ^{3} N\right)$ bandwidth blowup. This was followed by the statistically secure ORAM construction by Shi et al. [23], who introduced the tree-based paradigm. ORAM constructions in the tree-based paradigm have improved the bandwidth blowup from $O\left(\log ^{3} N\right)$ to $O\left(\log ^{2} N\right)$ [7, 22, 23, 25, 27. Though the computational assumptions have been removed, the statistically secure ORAMs still fail with a failure probability that is negligibly small in the number of data blocks stored in the ORAM.

Perfectly-secure ORAMs. Perfectly-secure ORAM was first studied by Damgård et al. [8]. Perfect security requires that a computationally unbounded server does not learn anything other than the number of requests with probability 1 . This implies that the oblivious program's memory access patterns should be identically distributed regardless of the inputs to the program; and thus with probability 1 , no information can be leaked about the secret inputs to the program. Damgård et al. 8 achieve an expected $O\left(\log ^{3} N\right)$ simulation overhead and $O(\log N)$ space blowup relative to the original RAM program. Raskin et al. 21] and Demertzis et al. 9] achieve a worst-case bandwidth blowup of $O\left(\sqrt{N} \frac{\log N}{\log \log N}\right)$ and $O\left(N^{1 / 3}\right)$, respectively. Chan et al. [5] improve upon Damgård et al.'s result [8] by avoiding the $O(\log N)$ blowup in space, and by showing a construction that is conceptually simpler. Our construction builds upon Chan et al. and improves the bandwidth blowup to worst-case $O\left(\log ^{2} N\right)$ while assuming three non-colluding servers.

Multi-server ORAMs. ORAMs in this category assume multiple non-colluding servers to improve bandwidth blowup 1, 15, 16, 18, 20. A comparison of the relevant schemes is presented in Table 1. Among these, the work that is closely related to ours is by Lu and Ostrovsky [20] which achieves a bandwidth blowup of $O(\log N)$ assuming two non-colluding servers. In their scheme, each server performs permutations for data that is stored by the other server. While their construction is computationally secure, we achieve perfect security for access patterns as well as the data itself. Moreover, our techniques can be used to perform an oblivious tight stable compaction and an oblivious merge operation in linear time; how to perform these operations in linear time were not known even for the computationally secure setting. On the other hand, our scheme achieves an $O\left(\log ^{2} N\right)$ bandwidth blowup and uses three servers. We remark that if we assume a perfectly secure encryption scheme, our construction can achieve perfectly secure access patterns using two servers. Abraham et al. 11, Gordon et al. [15] and Kushilevitz and Mour [18, construct multi-server ORAMs using PIR. Each of these constructions require the server to perform computation for using PIR operations. While Abraham et al. 1 achieve statistical security for access patterns, other work [15,18 is only computationally secure. While the work of Gordon et al. achieves a bandwidth blowup of $O(\log N)$, they require linear- 
Table 1: Comparison with existing multi-server Oblivious RAM schemes for block size $\Omega(\log N)$. All of the other schemes (including the statistically-secure schemes [1]) require two servers but assume the existence of an unconditionally secure encryption scheme. With a similar assumption, our work would indeed need only two servers too.

\begin{tabular}{|c|c|c|c|}
\hline Construction & $\begin{array}{c}\text { Bandwidth } \\
\text { Blowup }\end{array}$ & $\begin{array}{c}\text { Server } \\
\text { Computation }\end{array}$ & Security \\
\hline & & - & \\
\hline al. 15 & $O(\log N)$ & $O(N)$ & \\
\hline ushilevitz et & $O(\log N \cdot \omega(1))$ & $O(\log N \cdot \omega(1))$ & Computational \\
\hline Abraham et & $O\left(\log ^{2} N \cdot \omega(1)\right)$ & $O\left(\log ^{2} N \cdot \omega(1)\right)$ & Statistical \\
\hline ork & $O\left(\log ^{2} N\right)$ & - & Perfect \\
\hline
\end{tabular}

time server computation. Abraham et al. and Kushilevitz and Mour, on the other hand, are poly-logarithmic and logarithmic respectively, both in computation and bandwidth blowup. In comparison, our construction achieves perfect security and requires a passive server (i.e., a server that does not perform any computation) at a bandwidth blowup of $O\left(\log ^{2} N\right)$.

\section{Definitions}

In this section, we revisit how to define multi-server ORAM schemes for the case of semi-honest corruptions. Our definitions require that the adversary, controlling a subset of semi-honest corrupt servers, learns no secret information during the execution of the ORAM protocol. Specifically our adversary can observe all messages transmitted to and from corrupt servers, the rounds in which they were transmitted, as well as communication patterns between honest parties (including the client and honest servers). Our definition generalizes existing works [1] where they assume free encryption of data contents (even when statistical security is desired).

\subsection{Execution Model}

Protocol as a system of Interactive RAMs. We consider a protocol between multiple parties including a client, henceforth denoted by $\mathbf{C}$, and $k$ servers, denoted by $\mathbf{S}_{0}, \ldots, \mathbf{S}_{k-1}$, respectively. The client and all servers are Random Access Machines (RAMs) that interact with each other. Specifically, the client or each server has a CPU capable of computation and a memory that supports reads and writes; the CPU interacts with the memory to perform computation. The atomic unit of operation for memory is called a block. We assume that all RAMs can be probabilistic, i.e., they can read a random tape supplying a stream of random bits. 
Communication and timing. We assume pairwise channels between all parties. There are two notions of time in our execution model, CPU cycles and communication rounds. Without loss of generality, henceforth we assume that it takes the same amount of time compute each CPU instruction and to transmit each memory block over the network to another party (since we can always take the maximum of the two). Henceforth in this paper we often use the word round to denote the time that has elapsed since the beginning of the protocol.

Although we define RAMs on the servers as being capable of performing any arbitrary computation, all of our protocols require the servers to be passive, i.e., the server RAMs only perform read/write operations from the memory stored by it.

\subsection{Perfect Security under a Semi-Honest Adversary}

We consider the client to be trusted. The adversary can corrupt a subset of the servers (but it cannot corrupt the client) - although our constructions later secure against any individual corrupt server, we present definitions for the more general case, i.e., when the adversary can control more than one corrupt server.

We consider a semi-honest adversary, i.e., the corrupt servers still honestly follow the protocol; however, we would like to ensure that no undesired information will leak. To formally define security, we need to first define what the adversary can observe in a protocol's execution.

View of adversary view $^{\mathcal{A}}$. Suppose that the adversary $\mathcal{A}$ controls a subset of the servers - we abuse notation and use $\mathcal{A} \subset[k]$ to denote the set of corrupt servers. The view of the adversary, denoted by view ${ }^{\mathcal{A}}$ in a random run of the protocol consists of the following:

1. Corrupt parties' views, including 1) corrupt parties' inputs, 2) all randomness consumed by corrupt parties, and 3) an ordered sequence of all messages received by corrupt parties, including which party the message is received from, as well as the round in which each message is received. We assume that these messages are ordered by the round in which they are received, and then by the party from which it is received.

2. Honest communication pattern: when honest parties (including the client) exchange messages, the adversary observes their communication pattern: including which pairs of honest nodes exchange messages in which round.

We stress that in our model only one block can be exchanged between every pair in a round - thus the above view ${ }^{\mathcal{A}}$ definition effectively allows $\mathcal{A}$ to see the total length of messages exchanged between honest parties.

Remark 1. We remark that this definition captures a notion of timing patterns along with access patterns. For instance, suppose two servers store two sorted lists that needs to be merged. The client performs a regular merge operation to read from the two lists, reading the heads of the lists in each round. In such a scenario, depending on the rounds in which blocks are read from a server, an adversary that corrupts that server can compute the relative ordering of blocks between the two lists. 
Defining security in the ideal-real paradigm. Consider an ideal functionality $\mathcal{F}$ : upon receiving the input $\mathbf{I}_{0}$ from the client and inputs $\mathbf{I}_{1}, \ldots, \mathbf{I}_{k}$ from each of the $k$ servers respectively, and a random string $\rho$ sampled from some distribution, $\mathcal{F}$ computes

$$
\left(\mathbf{O}_{0}, \mathbf{O}_{1}, \ldots, \mathbf{O}_{k}\right):=\mathcal{F}\left(\mathbf{I}_{0}, \mathbf{I}_{1}, \ldots, \mathbf{I}_{k} ; \rho\right)
$$

where $\mathbf{O}_{0}$ is the client's output, and $\mathbf{O}_{1}, \ldots, \mathbf{O}_{k}$ denote the $k$ servers' outputs respectively.

Definition 1 (Perfect security in the presence of a semi-honest adversary). We say that "a protocol $\Pi$ perfectly securely realizes an ideal functionality $\mathcal{F}$ in the presence of a semi-honest adversary corrupting $t$ servers" iff for every adversary $\mathcal{A}$ that controls up to $t$ corrupt servers, there exists a simulator Sim such that for every input vector $\left(\mathbf{I}_{0}, \mathbf{I}_{1}, \ldots, \mathbf{I}_{k}\right)$, the following real- and ideal-world experiments output identical distributions:

- Ideal-world experiment. Sample $\rho$ at random and compute $\left(\mathbf{O}_{0}, \mathbf{O}_{1}, \ldots, \mathbf{O}_{k}\right):=$ $\mathcal{F}\left(\mathbf{I}_{0}, \mathbf{I}_{1}, \ldots, \mathbf{I}_{k}, \rho\right)$. Output the following tuple where we abuse notation and use $i \in \mathcal{A}$ to denote the fact that $i$ is corrupt:

$$
\operatorname{Sim}\left(\left\{\mathbf{I}_{i}, \mathbf{O}_{i}\right\}_{i \in \mathcal{A}}\right), \quad \mathbf{O}_{0},\left\{\mathbf{O}_{i}\right\}_{i \notin \mathcal{A}}
$$

- Real-world experiment. Execute the (possibly randomized) real-world protocol, and let $\mathbf{O}_{0}, \mathbf{O}_{1}, \ldots, \mathbf{O}_{k}$ be the outcome of the client and each of the $k$ servers respectively. Let view $^{\mathcal{A}}$ denote the view of the adversary $\mathcal{A}$ in this run. Now, output the following tuple:

$$
\operatorname{view}^{\mathcal{A}}, \mathbf{O}_{0},\left\{\mathbf{O}_{i}\right\}_{i \notin \mathcal{A}}
$$

Note that throughout the paper, we will define various building blocks that realize different ideal functionalities. The security of all building blocks can be defined in a unified approach with this paradigm. When we compose these building blocks to construct our full protocol, we can prove perfect security of the full protocol in a composable manner. By modularly proving the security of each building block, we can now think of each building block as interacting with an ideal functionality. This enables us to prove the security of the full protocol in the ideal world assuming the existence of these ideal functionalities.

Active-server vs. passive-server protocols. In active-server protocols, servers can perform arbitrary computation and send messages to each other. In passiveserver protocols, the servers act as passive memory and can only answer memory read and write requests from the client; furthermore, there is only client-server communication and servers do not communicate with each other. Obviously passive-server schemes are more general - in fact, all schemes in this paper are in the passive-server paradigm. We stress that all of our security definitions apply to both passive-server and active-server schemes. 


\subsection{Definition of $k$-Server Oblivious RAM}

Ideal logical memory. The ideal logical memory is defined in the most natural way. There is a memory array consisting of $N$ blocks where each block is $\Omega(\log N)$ bits long, and each block is identified by its unique address which takes value in the range $\{0,1, \ldots, N-1\}$.

Initially all blocks are set to 0 . Upon receiving (read, addr), the value of the block residing at address addr is returned. Upon receiving (write, addr, data), the block at address addr is overwritten with the data value data, and its old value (before being rewritten) is returned.

$k$-server ORAM. A $k$-server Oblivious RAM (ORAM) is a protocol between a client $\mathbf{C}$ and $k$ servers $\mathbf{S}_{1}, \ldots, \mathbf{S}_{k}$ which realizes an ideal logical memory. The execution of this protocol proceeds in a sequence of iterations: in each interaction, the client $\mathbf{C}$ receives a logical memory request of the form (read, addr) or (write, addr, data). It then engages in some (possibly randomized) protocol with the servers, at the end of which it produces some output thus completing the current iteration.

We require perfect correctness and perfect security as defined below. We refer to a sequence of logical memory requests as a request sequence for short.

- Perfect correctness. For any request sequence, with probability 1 , all of the client's outputs must be correct. In other words, we require that with probability 1 , all of the client's outputs must match what the an ideal logical memory would have output for the same request sequence.

- Perfect security under a semi-honest adversary. We say that a $k$-server ORAM scheme satisfies perfect security w.r.t. a semi-honest adversary corrupting $t$ servers, iff for every $\mathcal{A}$ that controls up to $t$ servers, and for every two request sequences $\mathbf{R}_{0}$ and $\mathbf{R}_{1}$ of equal length, the views view ${ }^{\mathcal{A}}\left(\mathbf{R}_{0}\right)$ and $\operatorname{view}^{\mathcal{A}}\left(\mathbf{R}_{1}\right)$ are identically distributed, where view ${ }^{\mathcal{A}}(\mathbf{R})$ denotes the view of $\mathcal{A}$ (as defined earlier in Section 2.2) under the request sequence $\mathbf{R}$.

Since we require perfect security (and our scheme is based on informationtheoretic secret-sharing), our notion resists adaptive corruptions and is composable.

\subsection{Resource Assumptions and Cost Metrics}

We assume that the client can store $O(1)$ blocks while the servers can store $O(N)$ blocks. We will use the metric bandwidth blowup to characterize the performance of our protocols. Bandwidth blowup is the (amortized) number of blocks queried in the ORAM simulation to query a single virtual block. We also note that since the servers do not perform any computation, and the client always performs an $O(1)$ computation on its $O(1)$ storage, an $O(X)$ bandwidth blowup also corresponds to an $O(X)$ runtime for our protocol. 


\section{Core Building Blocks: Definitions and Constructions}

Imagine that there are three servers denoted $\mathbf{S}_{0}, \mathbf{S}_{1}$, and $\mathbf{S}_{2}$, and a client denoted C. We use $\mathbf{S}_{b}, b \in \mathbb{Z}_{3}$ to refer to a specific server. Arithmetic performed on the subscript $b$ is done modulo 3 .

\subsection{Useful Definitions}

Let $T$ denote a list of blocks where each block is either a real block containing a payload string and a logical address; or a dummy block denoted $\perp$. We define sorted and semi-sorted as follows:

- Sorted: T is said to be sorted iff all real blocks appear before dummy ones; and all the real blocks appear in increasing order of their logical addresses. If multiple blocks have the same logical address, their relative order can be arbitrary.

- Semi-sorted: T is said to be semi-sorted iff all the real blocks appear in increasing order of their logical addresses, and ties may be broken arbitrarily. However, the real blocks are allowed to be interspersed by dummy blocks.

Array Notation. We assume each location of an array T stores a block which is a bit-string of length $B$. Given two arrays $T_{1}$ and $T_{2}$, we use $T_{1} \oplus T_{2}$ to denote the resulting array after performing bitwise-XOR on the corresponding elements at each index of the two arrays; if the two arrays are of different lengths, we assume the shorter array is appended with a sufficient number of zero elements.

Permutation Notation. When a permutation $\pi:[n] \rightarrow[n]$ is applied to an array T indexed by $[n]$ to produce $\pi(\mathrm{T})$, we mean the element currently at location $i$ will be moved to location $\pi(i)$. When we compose permutations, $\pi \circ \sigma$ means that $\pi$ is applied before $\sigma$. We use $\mathfrak{e}$ to denote the identity permutation.

Layout. A layout is a way to store some data $T$ on three servers such that the data can be recovered by combining information on the three servers. Recall that the client has only $O(1)$ blocks of space, and our protocol does not require that the client stores any persistent data.

Whenever some data $T$ is stored on a server, informally speaking, we need to ensure two things: 1) The server does not learn the data $T$ itself, and 2) The server does not learn which index $\mathrm{i}$ of the data is accessed. In order to ensure the prior, we XOR secret-share the data $T:=\mathrm{T}_{0} \oplus \mathrm{T}_{1} \oplus \mathrm{T}_{2}$ between three servers $\mathbf{S}_{b}, b \in \mathbb{Z}_{3}$ such that $\mathbf{S}_{b}$ stores $\mathbf{T}_{b}$. For a server to not learn which index $i$ in $\mathrm{T}$ is accessed, we ensure that the data is permuted, and the access happens to the permuted data. If the data is accessed on the same server that permutes the data, then the index $i$ will still be revealed. Thus, for each share $\mathrm{T}_{b}$, we ensure

that one server permutes it and we access it from another server, i.e., we have two types of servers: 
- Each server $\mathbf{S}_{b}$ acts as a storage server for the $b$-th share, and thus it knows $\mathrm{T}_{b}$.

- Each server $\mathbf{S}_{b}$ also acts as the permutation server for the $(b+1)$-th share, and thus it also knows $\mathrm{T}_{b+1}$ as well as $\pi_{b+1}$.

Throughout the paper, a layout is of the following form

$$
\text { 3-server layout : } \quad\left\{\pi_{b}, \mathrm{~T}_{b}\right\}_{b \in \mathbb{Z}_{3}}
$$

where $\mathbf{T}_{b}$ and $\left(\pi_{b+1}, \mathbf{T}_{b+1}\right)$ are stored by server $\mathbf{S}_{b}$. As mentioned, $\mathbf{S}_{b}$ not only knows its own share $\left(\mathrm{T}_{b}\right)$ but also the permutation and share of the next server $\left(\pi_{b+1}, \mathrm{~T}_{b+1}\right)$.

Specifically, $\mathrm{T}_{0}, \mathrm{~T}_{1}, \mathrm{~T}_{2}$ denote lists of blocks of equal length: we denote $n=$ $\left|\mathrm{T}_{0}\right|=\left|\mathrm{T}_{1}\right|=\left|\mathrm{T}_{2}\right|$. Further, $\pi_{b+1}:[n] \rightarrow[n]$ is a permutation stored by server $\mathbf{S}_{b}$ for the list $T_{b+1}$. Unless there is ambiguity, we use $\oplus_{b}$ to mean applying $\oplus_{b \in \mathbb{Z}_{3}}$ to three underlying arrays.

The above layout is supposed to store the array that can be recovered by:

$$
\oplus_{b} \pi_{b}^{-1}\left(\mathrm{~T}_{b}\right)
$$

Henceforth, given a layout $\left\{\pi_{b}, \mathrm{~T}_{b}\right\}_{b \in \mathbb{Z}_{3}}$, we say that the layout is sorted (or semi-sorted) iff $\oplus_{b} \pi_{b}^{-1}\left(\mathrm{~T}_{b}\right)$ is sorted (or semi-sorted).

Special Case. Sometimes the blocks secret-shared among $\mathbf{S}_{0}, \mathbf{S}_{1}, \mathbf{S}_{2}$ may be unpermuted, i.e., for each $b \in \mathbb{Z}_{3}, \pi_{b}$ is the identity permutation $\mathfrak{e}$. In this case, the layout is

$$
\text { Unpermuted layout : } \quad\left\{\mathfrak{e}, \mathrm{T}_{b}\right\}_{b \in \mathbb{Z}_{3}}
$$

For brevity, the unpermuted layout $\left\{\mathfrak{e}, \mathrm{T}_{b}\right\}_{b \in \mathbb{Z}_{3}}$ is also denoted by the abstract array $\mathrm{T}$.

Definition 2 (Secret Write). An abstract array $\mathrm{T}$ corresponds to some unpermuted layout $\left\{\mathfrak{e}, \mathrm{T}_{b}\right\}_{b \in \mathbb{Z}_{3}}$. We say that the client secretly writes a value $\mathrm{B}$ to the array $\mathrm{T}$ at index $i$, when it does the following:

- Sample random values $\mathrm{B}_{0}$ and $\mathrm{B}_{1}$ independently, and compute $\mathrm{B}_{2}:=\mathrm{B} \oplus$ $\mathrm{B}_{0} \oplus \mathrm{B}_{1}$.

- For each $b \in \mathbb{Z}_{3}$, the client writes $\mathrm{T}_{b}[i]:=\mathrm{B}_{b}$ on server $\mathbf{S}_{b}$ (and $\mathbf{S}_{b-1}$ ).

Definition 3 (Reconstruct). Given some layout $\left\{\pi_{b}, \mathrm{~T}_{b}\right\}_{b \in \mathbb{Z}_{3}}$, the client reconstructs a value from using tuple $\left(i_{0}, i_{1}, i_{2}\right)$ of indices, when it does the following:

- For each $b \in \mathbb{Z}_{3}$, the client reads $\mathrm{T}_{b}\left[i_{b}\right]$ from server $\mathbf{S}_{b}$. (It is important that the client reads $\mathrm{T}_{b}$ from $\mathbf{S}_{b}$, even though $\mathrm{T}_{b}$ is stored in both $S_{b}$ and $S_{b-1}$.)

- The reconstructed value is $\oplus_{b} \mathrm{~T}_{b}\left[i_{b}\right]$.

Protocol Notation. All protocols are denoted as out $\leftarrow \operatorname{Prot}(\sin$, cin). Here, sin and cin are respectively server and client inputs to the protocol Prot. Except for in an ORAM Lookup, all the outputs out are sent to the server. 


\section{$3.2 \quad$ Permute and Unpermute}

Non-oblivious random permutation. Fisher and Yates [10] show how to generate a uniformly random permutation $\pi:[n] \rightarrow[n]$ in $O(n)$ time steps. This implies that the client can write a random permutation on a server with $O(n)$ bandwidth. The permutation is non-oblivious, i.e., the server does learn the permutation generated.

Definition of Permute. Permute is a protocol that realizes an ideal functionality $\mathcal{F}_{\text {perm }}$ as defined below. Intuitively, this functionality takes some unpermuted input layout (i.e., unpermuted secret-shared inputs) and three additional permutations $\pi_{b+1}$ from the three permutation servers $\mathbf{S}_{b}$. The functionality produces an output such that the three shares are secret-shared again, and the share received by storage server $\mathbf{S}_{b+1}$ is permuted using $\pi_{b+1}$. Secret-sharing the data again before applying the new permutations ensures that a storage server $\mathbf{S}_{b+1}$ does not learn the permutation $\pi_{b+1}$ applied to its share.

- $\left\{\pi_{b}, \mathbf{T}_{b}^{\prime}\right\}_{b \in \mathbb{Z}_{3}} \leftarrow$ Permute $\left(\left(\left\{\mathfrak{e}, \mathbf{T}_{\mathbf{b}}\right\}_{\mathbf{b} \in \mathbb{Z}_{3}},\left\{\pi_{\mathbf{b}}\right\}_{\mathbf{b} \in \mathbb{Z}_{3}}\right), \perp\right):$

- Input: Let $\left\{\mathfrak{e}, \mathrm{T}_{b}\right\}_{b \in \mathbb{Z}_{3}}$ be the unpermuted layout provided as input. (Recall that $\mathrm{T}_{b}$ and $\mathrm{T}_{b+1}$ are stored in server $\mathbf{S}_{b}$.)

Moreover, for each $b \in \mathbb{Z}_{3}, \mathbf{S}_{b}$ has an additional permutation $\pi_{b+1}$ as input (which could be generated by the client for instance).

The arrays have the same length $\left|\mathrm{T}_{0}\right|=\left|\mathrm{T}_{1}\right|=\left|\mathrm{T}_{2}\right|=n$, for some $n$. The client obtains $\perp$ as the input.

- Ideal functionality $\mathcal{F}_{\text {perm }}$ :

Sample independently and uniformly random $\widehat{T}_{0}, \widehat{T}_{1}$ of length $n$. Now, define $\widehat{T}_{2}:=\widehat{T}_{0} \oplus \widehat{T}_{1} \oplus\left(\oplus_{b} T_{b}\right)$, i.e., $\oplus_{b} \widehat{T}_{b}=\oplus_{b} T_{b}$.

For each $b \in \mathbb{Z}_{3}$, define $\mathbf{T}_{b}^{\prime}:=\pi_{b}\left(\widehat{T}_{b}\right)$.

The output layout is $\left\{\pi_{b}, \mathbf{T}_{b}^{\prime}\right\}_{b \in \mathbb{Z}_{3}}$, and the client's output is $\perp$.

Protocol Permute. The implementation of $\mathcal{F}_{\text {perm }}$ proceeds as follows:

1. Mask shares. For each data block, the client first generates block "masks" that sum up to zero, and then applies mask to $T_{b+1}$ on server $\mathbf{S}_{b}$. Specifically, the client does the following, for each $i \in[n]$ :

- Generate block "masks" that sum up to zero, i.e., sample independent random blocks $\mathrm{B}_{0}^{i}$ and $\mathrm{B}_{1}^{i}$, and compute $\mathrm{B}_{2}^{i}:=\mathrm{B}_{0}^{i} \oplus \mathrm{B}_{1}^{i}$.

- Apply mask $\mathrm{B}_{b+1}^{i}$ to $\mathbf{T}_{b+1}[i]$ stored on server $\mathbf{S}_{b}$, i.e., for each $i \in[b]$, the client writes $\widehat{\mathrm{T}}_{b+1}[i] \leftarrow \mathrm{T}_{b+1}[i] \oplus \mathrm{B}_{b+1}^{i}$ on server $\mathbf{S}_{b}$.

2. Permute share of $\mathbf{S}_{b+1}$ and send result to $\mathbf{S}_{b+1}$. The client uses $\pi_{b+1}$ to permute a share on the permutation server and then sends this permuted share to the storage server, i.e., for each $b \in \mathbb{Z}_{3}$, the client computes computes $\mathbf{T}_{b+1}^{\prime}:=\pi_{b+1}\left(\widehat{\mathbf{T}}_{b+1}\right)$ on server $\mathbf{S}_{b}$, and sends the result $\mathbf{T}_{b+1}^{\prime}$ to $\mathbf{S}_{b+1}$. Each server $\mathbf{S}_{b}$ stores $\mathbf{T}_{b}^{\prime}$ and $\left(\pi_{b+1}, \mathbf{T}_{b+1}^{\prime}\right)$; hence, the new layout $\left\{\pi_{b}, \mathbf{T}_{b}^{\prime}\right\}_{b \in \mathbb{Z}_{3}}$ is achieved. 
Theorem 3. The Permute protocol perfectly securely realizes the ideal functionality $\mathcal{F}_{\text {perm }}$ in the presence of a semi-honest adversary corrupting a single server with $O(n)$ bandwidth.

Proof. By construction, the implementation of the protocol applies the correct permutation on each server's array and re-distributes the secret shares using fresh independent randomness. Hence, the marginal distribution of the protocol's outputs is exactly the same as that of the ideal functionality.

Fix some $b \in \mathbb{Z}_{3}$ and consider the view ${ }^{b}$ of the corrupt server $\mathbf{S}_{b}$. Since the leakage of $\mathcal{F}_{\text {perm }}$ is empty, we will in fact show that given the inputs and the outputs to server $\mathbf{S}_{b}$, the view ${ }^{b}$ is totally determined and has no more randomness. Hence, given $\mathbf{I}_{b}$ and conditioning on $\mathbf{O}_{b}$, the view ${ }^{b}$ is trivially independent of the outputs of the client and other servers.

Then, given the inputs $\mathbf{I}_{b}$ and the outputs $\mathbf{O}_{b}$ to $\mathbf{S}_{b}$, a simulator simply returns the view of $\mathbf{S}_{b}$ uniquely determined by $\mathbf{I}_{b}$ and $\mathbf{O}_{b}$.

The inputs to $\mathbf{S}_{b}$ are the arrays $\mathbf{T}_{b}$ and $\mathbf{T}_{b+1}$, and the permutation $\pi_{b+1}$. The outputs are the arrays $\mathrm{T}_{b}^{\prime}$ and $\mathrm{T}_{b+1}^{\prime}$, and also the permutation $\pi_{b+1}$. We next consider each part of view ${ }^{b}$.

1. Communication Pattern. The communication pattern between the client and all the servers only depends on $n$.

2. Data Structure. We next analyze the intermediate data that is observed by $\mathbf{S}_{b}$. The arrays $\mathbf{T}_{b}^{\prime}$ and $\mathbf{T}_{b+1}^{\prime}$ are in $\mathbf{S}_{b}$ 's outputs. Hence, it suffices to consider the intermediate array $\widehat{\mathbf{T}}_{b+1}=\pi_{b+1}^{-1}\left(\mathbf{T}_{b}^{\prime}\right)$, which is totally determined by the outputs.

We have shown that the view ${ }^{b}$ is actually a deterministic function of the inputs and the outputs of $\mathbf{S}_{b}$, as required.

Efficiency. Recall that it takes $O(1)$ time to process one block. From the construction, it is straightforward that linear scans are performed on the relevant arrays.

In particular, the two steps - masking shares and permuting the shares - can be done with $O(n)$ bandwidth. Moreover, the client can generate a permutation on the server with $O(n)$ bandwidth, when each block has $\Omega(\log n)$ bits, using the Fisher-Yates algorithm [10.

Definition of Unpermute. Unpermute is a protocol that realizes an ideal functionality $\mathcal{F}_{\text {unperm }}$ as defined below. Intuitively, this functionality reverses the effect of $\mathcal{F}_{\text {perm. }}$. It takes some permuted input layout, and returns the corresponding unpermuted layout. However, to avoid each server knowing its original permutation, the contents of each entry needs to be secret-shared again.

$-\left\{\mathfrak{e}, \mathrm{T}_{b}^{\prime}\right\}_{b \in \mathbb{Z}_{3}} \leftarrow$ Unpermute $\left(\left\{\pi_{\mathrm{b}}, \mathrm{T}_{\mathrm{b}}\right\}_{\mathrm{b} \in \mathbb{Z}_{3}}, \perp\right):$ 
- Input: Let $\left\{\pi_{b}, \mathbf{T}_{b}\right\}_{b \in \mathbb{Z}_{3}}$ be the layout provided as input. (Recall that $\mathbf{T}_{b}$ and $\left(\pi_{b+1}, \mathbf{T}_{b+1}\right)$ are stored in server $\mathbf{S}_{b}$.)

The arrays have the same length $\left|\mathrm{T}_{0}\right|=\left|\mathrm{T}_{1}\right|=\left|\mathrm{T}_{2}\right|=n$, for some $n$. The client obtains $\perp$ as the input.

- Ideal functionality $\mathcal{F}_{\text {unperm: }}$ :

Sample independently and uniformly random $\mathrm{T}^{\prime}{ }_{0}, \mathrm{~T}^{\prime}{ }_{1}$ of length $n$.

Now, define $T_{2}^{\prime}:=T_{0}^{\prime} \oplus T_{1}^{\prime} \oplus\left(\oplus_{b} \pi_{b}^{-1}\left(T_{b}\right)\right)$, i.e., $\oplus_{b} T_{b}^{\prime}=\oplus_{b} \pi_{b}^{-1}\left(T_{b}\right)$.

The output layout is $\left\{\mathfrak{e}, \mathbf{T}_{b}^{\prime}\right\}_{b \in \mathbb{Z}_{3}}$, and the client's output is $\perp$.

Protocol Unpermute. The implementation of $\mathcal{F}_{\text {unperm }}$ proceeds as follows:

1. Compute inverse permutations. For each $b \in \mathbb{Z}_{3}$, the client computes the inverse permutation $\widehat{T}_{b+1}:=\pi_{b+1}^{-1}\left(\mathbf{T}_{b+1}\right)$ on server $\mathbf{S}_{b}$.

2. Mask shares. For each data block, the client generates block "masks" that sum up to zero and then applies the mask to $\mathbf{T}_{b+1}$ on server $\mathbf{S}_{b}$. Specifically, the client performs the following, for each $i \in[n]$ :

- Generate block "masks" that sum up to zero, i.e., Sample independent random blocks $\mathrm{B}_{0}^{i}$ and $\mathrm{B}_{1}^{i}$, and compute $\mathrm{B}_{2}^{i}:=\mathrm{B}_{0}^{i} \oplus \mathrm{B}_{1}^{i}$.

- Apply mask $\mathrm{B}_{b+1}^{i}$ to $\mathrm{T}_{b+1}[i]$ stored on server $\mathbf{S}_{b}$, i.e., for each $i \in[b]$, the client writes $\mathrm{T}_{b+1}^{\prime}[i] \leftarrow \widehat{\mathrm{T}}_{b+1}[i] \oplus \mathrm{B}_{b+1}^{i}$ on server $\mathbf{S}_{b}$.

3. For each $b \in \mathbb{Z}_{3}$, the server $\mathbf{S}_{b}$ sends $\mathbf{T}_{b+1}^{\prime}$ to $\mathbf{S}_{b+1}$.

Hence, the new layout $\left\{\mathfrak{e}, \mathbf{T}_{b}^{\prime}\right\}_{b \in \mathbb{Z}_{3}}$ is achieved.

Theorem 4. The Unpermute protocol perfectly securely realizes the ideal functionality $\mathcal{F}_{\text {unperm }}$ in the presence of a semi-honest adversary corrupting a single server with $O(n)$ bandwidth blowup.

Proof. The proof is essentially the same as Theorem 3. It can be checked that for each $b \in \mathbb{Z}_{3}$, the view ${ }^{b}$ is a deterministic function of the inputs and the outputs of $\mathbf{S}_{b}$.

\subsection{Stable Compaction}

Definition of StableCompact. StableCompact is a protocol that realizes an ideal functionality $\mathcal{F}_{\text {compact }}$, as defined below:

$-\left\{\mathfrak{e}, \mathrm{T}_{b}^{\prime}\right\}_{b \in \mathbb{Z}_{3}} \leftarrow$ StableCompact $\left(\left\{\mathfrak{e}, \mathrm{T}_{b}\right\}_{b \in \mathbb{Z}_{3}}, \perp\right)$ :

- Input layout: A semi-sorted, unpermuted layout denoted $\left\{\mathfrak{e}, T_{b}\right\}_{b \in \mathbb{Z}_{3}}$.

- Ideal functionality $\mathcal{F}_{\text {compact }}: \mathcal{F}_{\text {compact }}$ computes $\mathrm{T}^{*}:=\mathrm{T}_{0} \oplus \mathrm{T}_{1} \oplus \mathrm{T}_{2}$; it then moves all dummy blocks in $T^{*}$ to the end of the array, while keeping the relative order of real blocks unchanged.

Now, $\mathcal{F}_{\text {compact }}$ randomly samples $\mathrm{T}_{0}^{\prime}, \mathrm{T}_{1}^{\prime}$ of appropriate length and computes $T_{2}^{\prime}$ such that $T^{*}=T_{0}^{\prime} \oplus T_{1}^{\prime} \oplus T_{2}^{\prime}$. The output layout is a sorted, unpermuted layout $\left\{\mathfrak{e}, \mathrm{T}_{b}^{\prime}\right\}_{b \in \mathbb{Z}_{3}}$. 
StableCompact Protocol. The input is a semi-sorted, unpermuted layout, and we would like to turn it into a sorted, unpermuted layout obliviously. The key idea is to permute each share of the list (stored on the 3 servers respectively), such that the storage server for each share does not know the permutation. Now, the client accesses all real elements in a sorted order, and then accesses all dummy elements, writing down the elements in a secret-shared manner as the accesses are made. We can achieve this if each real or dummy element is tagged with a pointer to its next element, and the pointer is in fact a 3-tuple that is also secret shared on the 3 servers - each element in the 3-tuple indicates where the next element is in one of the 3 permutations.

Therefore, the crux of the algorithm is to tag each (secret-shared) element with a (secret-shared) position tuple, indicating where its next element is - this will effectively create two linked list structures (one for real and one for dummy): each element in the linked lists is secret shared in to 3 shares, and each share resides on its storage server at an independent random location.

The detailed protocol is as follows:

1. First, each server $\mathbf{S}_{b}$ acts as the permutation server for $\mathbf{S}_{b+1}$. Thus, the client generates a random permutation $\pi_{b+1}$ on the permutation server $\mathbf{S}_{b}$ using the Fisher-Yates algorithm described in Section 3.2. Basically, for each index $i$ of the original list the client writes down, on each $\mathbf{S}_{b}$, that its $(b+1)$-th share (out of 3 shares), wants to be in position $\pi_{b+1}(i)$.

2. Next, the client makes a reverse scan of $\left(\mathrm{T}_{0}, \pi_{0}\right),\left(\mathrm{T}_{1}, \pi_{1}\right),\left(\mathrm{T}_{2}, \pi_{2}\right)$ for $i=n$ down to 1 . The client can access $\left(\mathbf{T}_{b+1}[i], \pi_{b+1}(i)\right)$ by talking to $\mathbf{S}_{b}$. In this reverse scan, the client always locally remembers the position tuple of the last real element encountered (henceforth denoted $\mathfrak{p}_{\text {real }}$ ) and the position tuple of the last dummy element encountered (henceforth denoted $\mathfrak{p}_{\text {dummy }}$ ). Thus, if $\mathbf{T}\left[k_{\text {real }}\right]$ is the last seen real element, then the client remembers $\mathfrak{p}_{\text {real }}=\left(\pi_{b}\left(k_{\text {real }}\right): b \in \mathbb{Z}_{3}\right) \cdot \mathfrak{p}_{\text {dummy }}$ is updated analogously. Initially, $\mathfrak{p}_{\text {real }}$ and $\mathfrak{p}_{\text {dummy }}$ are set to $\perp$.

During this scan, whenever a real element $\mathrm{T}[i]$ is encountered, the client secretly writes the link $\mathrm{L}[i]:=\mathfrak{p}_{\text {real }}$, i.e., $\mathrm{L}[i]$ represents secret shares of the next pointers for the real element and $L$ itself represents an abstract linked list of real elements. The links for dummy elements are updated analogously using $\mathfrak{p}_{\text {dummy }}$.

At the end of this reverse scan, the client remembers the position tuple for the first real of the linked list denoted $\mathfrak{p}_{\text {real }}^{1}$ and position tuple for the first dummy denoted $\mathfrak{p}_{\text {dummy }}^{1}$.

3. Next, we call Permute inputting 1) the original layout - but importantly, now each element is tagged with a position tuple (that is also secret shared); and 2 ) the three permutations chosen by each $\mathbf{S}_{b}$ (acting as the permutation server for $\left.\mathbf{S}_{b+1}\right)$. Thus, Permute is applied to the combined layout $\left\{\mathfrak{e},\left(\mathrm{T}_{b}, \mathrm{~L}_{b}\right)\right\}_{b \in \mathbb{Z}_{3}}$, where $\mathbf{S}_{b}$ has input permutation $\pi_{b+1}$. Let the output of Permute be denoted by $\left\{\pi_{b},\left(\mathrm{~T}_{b}^{\prime}, \mathrm{L}_{b}^{\prime}\right)\right\}_{b \in \mathbb{Z}_{3}}$.

4. Finally, the client traverses first the real linked list (whose start position tuple is $\mathfrak{p}_{\text {real }}^{1}$ ) and then the dummy linked list (whose start position tuple is $\mathfrak{p}_{\text {dummy }}^{1}$ ). 
During this traversal, the client secretly writes each element encountered to produce the sorted and unpermuted output layout.

More precisely, the client secretly writes an abstract array $\mathrm{T}^{\prime \prime}$ element by element. Start with $k \leftarrow 0$ and $\mathfrak{p} \leftarrow \mathfrak{p}_{\text {real }}^{1}$.

The client reconstructs element $\mathrm{B}:=\oplus \mathrm{T}_{b}^{\prime}\left[\mathfrak{p}_{b}\right]$ and the next pointer of the linked list next $:=\oplus \mathrm{L}_{b}^{\prime}\left[\mathfrak{p}_{b}\right]$; the client secretly writes to the abstract array $\mathrm{T}^{\prime \prime}[k]:=\mathrm{B}$.

Then, it updates $k \leftarrow k+1$ and $\mathfrak{p} \leftarrow$ next, and continues to the next element; if the end of the real list is reached, then it sets $\mathfrak{p} \leftarrow \mathfrak{p}_{\text {dummy }}^{1}$. This continues until the whole (abstract) $T^{\prime \prime}$ is secretly written to the three servers.

5. The new layout $\left\{\mathfrak{e}, T_{b}^{\prime \prime}\right\}_{b \in \mathbb{Z}_{3}}$ is constructed.

Theorem 5. The StableCompact protocol perfectly securely realizes the ideal functionality $\mathcal{F}_{\text {compact }}$ in the presence of a semi-honest adversary corrupting a single server with $O(n)$ bandwidth.

Proof. By construction, the protocol correctly removes dummy elements and preserves the original order of real elements, where the secret shares are redistributed using independent randomness. Hence, the marginal distribution on the outputs is the same for both the protocol and the ideal functionality.

We fix the inputs of all servers, and some $b \in \mathbb{Z}_{3}$. The goal is to show that (1) the view ${ }^{b}$ follows a distribution that is totally determined by the inputs $\mathbf{I}_{b}$ and the outputs $\mathbf{O}_{b}$ of the corrupt $\mathbf{S}_{b} ;(2)$ conditioning on $\mathbf{O}_{b}$, view ${ }^{b}$ is independent of the outputs of the client and all other servers.

The second part is easy, because the inputs are fixed. Hence, conditioning on $\mathbf{O}_{b}$ (which includes $\mathbf{T}_{b}^{\prime \prime}$ and $\mathbf{T}_{b+1}^{\prime \prime}$ ), $\mathbf{T}_{b+2}^{\prime \prime}$ has no more randomness and totally determines the outputs of other servers.

To prove the first part, our strategy is to decompose view ${ }^{b}$ into a list components, and show that fixing $\mathbf{I}_{b}$ and conditioning on $\mathbf{O}_{b}$ and a prefix of the components, the distribution of the next component can be determined. Hence, this also gives the definition of a simulator.

First, observe that in the last step, the client re-distributes the shares, and gives output $\mathbf{O}_{b}$ to $\mathbf{S}_{b}$; moreover, the shares of $\mathbf{T}^{\prime \prime}$ are generated with fresh independent randomness. Hence, the distribution of the part of view ${ }^{b}$ excluding $\mathbf{O}_{b}$ is independent of $\mathbf{O}_{b}$. We consider the components of view ${ }^{b}$ in the following order, which is also how a simulator generates a view after seeing $\mathbf{I}_{b}$.

1. Communication Pattern. Observe that from the description of the algorithm, the communication pattern between the client and the servers depends only on the length $n$ of the input array.

2. Random permutation $\pi_{b+1}$. This is independently generated using fresh randomness.

3. Link $\mathrm{L}$ creation. The (abstract) array $\mathrm{L}$ is created by reverse linear scan. The shares $L_{b}$ and $L_{b+1}$ received by $\mathbf{S}_{b}$ are generated by fresh independent randomness.

4. Permute subroutine. By the correctness of the Permute, the shares of the outputs $\left(\mathbf{T}^{\prime}, \mathrm{L}^{\prime}\right)$ received by $\mathbf{S}_{b}$ follow an independent and uniform random 
distribution. By the perfect security of Permute, the component of view ${ }^{b}$ due to Permute depends only on the inputs (which include the shares of $T$ and L) and the outputs of the subroutine.

5. List traversal. Since $\mathbf{S}_{b}$ does not know $\pi_{b}$ (which is generated using independent randomness by $\mathbf{S}_{b-1}$ ), from $\mathbf{S}_{b}$ 's point of view, its array $\mathbf{T}_{b}^{\prime}$ is traversed in an independent and uniform random order.

Therefore, we have described a simulator procedure that samples the view ${ }^{b}$ step-by-step, given $\mathbf{I}_{b}$ and $\mathbf{O}_{b}$.

Efficiency. Each of the steps in the protocol can be executed with a bandwidth of $O(n)$. Step 1 can be performed using Fisher-Yates shuffle algorithm. In steps 2 and 4, the client linearly scans the abstract lists $\mathrm{T}, \mathrm{T}^{\prime \prime}$ and the links $\mathrm{L}, \mathrm{L}^{\prime}$. Accessing each array costs $O(n)$ bandwidth. Finally, step 3 invokes permute, which requires $O(n)$ bandwidth (Theorem 3 ).

\subsection{Merging}

Definition of Merge. Merge is a protocol that realizes an ideal functionality $\mathcal{F}_{\text {merge }}$ as defined below:

$-\left\{\mathfrak{e}, \mathrm{U}_{b}^{\prime \prime}\right\}_{b \in \mathbb{Z}_{3}} \leftarrow \operatorname{Merge}\left(\left\{\mathfrak{e},\left(\mathrm{T}_{\mathrm{b}}, \mathrm{T}_{\mathrm{b}}^{\prime}\right)\right\}_{\left.\mathrm{b} \in \mathbb{Z}_{3}, \perp\right):}\right.$

- Input layout: Two semi-sorted, unpermuted layouts denoted $\left\{\mathfrak{e}, \mathrm{T}_{b}\right\}_{b \in \mathbb{Z}_{3}}$ and $\left\{\mathfrak{e}, \mathbf{T}_{b}^{\prime}\right\}_{b \in \mathbb{Z}_{3}}$ denoting abstract lists $\mathrm{T}$ and $\mathrm{T}^{\prime}$, where all the arrays have the same length $n$.

- Ideal functionality $\mathcal{F}_{\text {merge }}$ : First, $\mathcal{F}_{\text {merge }}$ merges the two lists $\mathrm{T}_{0} \oplus \mathrm{T}_{1} \oplus \mathrm{T}_{2}$ and $\mathrm{T}_{0}^{\prime} \oplus \mathrm{T}_{1}^{\prime} \oplus \mathrm{T}_{2}^{\prime}$, such that the resulting array is sorted with all dummy blocks at the end. Let $U^{\prime \prime}$ be this merged result. Now, $\mathcal{F}_{\text {merge }}$ randomly samples $\mathrm{U}_{0}^{\prime \prime}$ and $\mathrm{U}_{1}^{\prime \prime}$ independently of appropriate length and computes $\mathrm{U}_{2}^{\prime \prime}$ such that $\mathrm{U}^{\prime \prime}=\mathrm{U}_{0}^{\prime \prime} \oplus \mathrm{U}_{1}^{\prime \prime} \oplus \mathrm{U}_{2}^{\prime \prime}$. The output layout is a sorted, unpermuted layout $\left\{\mathfrak{e}, \mathrm{U}_{b}^{\prime \prime}\right\}_{b \in \mathbb{Z}_{3}}$.

Merge Protocol. The protocol receives as input, two semi-sorted, unpermuted layouts and produces a merged, sorted, unpermuted layout as the output. The key idea is to permute the concatenation of the two semi-sorted inputs such that the storage servers do not know the permutation. Now, the client accesses real elements in both lists in the sorted order using the storage servers to produce a merged output. Given that a concatenation of the lists is permuted together, elements from which list is accessed is not revealed during the merge operation, thereby allowing us to merge the two lists obliviously. In order to access the two lists in a sorted order, the client creates a linked list of real and dummy elements using the permutation servers, similar to the StableCompact protocol in Section 3.3 .

The detailed protocol works as follows: 
1. First, the client concatenates the two abstract lists $T$ and $T^{\prime}$ to obtain an abstract list $\mathrm{U}$ of size $2 n$, i.e., we interpret $\mathrm{U}_{b}$ as the concatenation of $\mathrm{T}_{b}$ and $\mathrm{T}_{b}^{\prime}$ for each $b \in \mathbb{Z}_{3}$. Specifically, $\mathrm{U}_{b}[0, n-1]$ corresponds to $\mathrm{T}_{b}$ and $\mathrm{U}_{b}[n, 2 n-1]$ corresponds to $\mathrm{T}_{b}^{\prime}$.

2. Now, each server $\mathbf{S}_{b}$ acts as the permutation server for $\mathbf{S}_{b+1}$. The client generates a random permutation $\pi_{b+1}:[2 n] \rightarrow[2 n]$ on server $\mathbf{S}_{b+1}$ using the Fisher-Yates algorithm described in Section 3.2 $\pi_{b+1}(i)$ represents the position of the $(b+1)$-th share and is stored on server $\mathbf{S}_{b}$.

3. The client now performs a reverse scan of $\left(\mathrm{U}_{0}, \pi_{0}\right),\left(\mathrm{U}_{1}, \pi_{1}\right),\left(\mathrm{U}_{2}, \pi_{2}\right)$ for $i=n$ down to 1 . During this reverse scan, the client always locally remembers the position tuples of the last real element and last dummy element encountered for both the lists. Let them be denoted by $\mathfrak{p}_{\text {real }}, \mathfrak{p}_{\text {real }}^{\prime}, \mathfrak{p}_{\text {dummy }}$, and $\mathfrak{p}_{\text {dummy }}^{\prime}$. Thus, if $\mathrm{U}\left[k_{\text {real }}\right]$ is the last seen real element from the first list, the client remembers $\mathfrak{p}_{\text {real }}=\left(\pi_{b}\left(k_{\text {real }}\right): b \in \mathbb{Z}_{3}\right)$. The other position tuples are updated analogously. Each of these tuples are initially set to $\perp$.

During the reverse scan, the client maintains an abstract linked list $L$ in the following manner. When $\mathrm{U}[i]$ is processed, if it is a real element from the first list, then the client secretly writes the link $\mathrm{L}[i]:=\mathfrak{p}_{\text {real }} . \mathrm{L}[i]$ represents secret shares of the next pointers for a real element from the first list. The cases for $\mathfrak{p}_{\text {real }}^{\prime}, \mathfrak{p}_{\text {dummy }}$, and $\mathfrak{p}_{\text {dummy }}^{\prime}$ are analogous.

At the end of this reverse scan, the client remembers the position tuple for the first real and first dummy elements of both linked lists. They are denoted by $\mathfrak{p}_{\text {real }}^{1}, \mathfrak{p}_{\text {real }}^{\prime 1}, \mathfrak{p}_{\text {dummy }}^{1}$, and $\mathfrak{p}_{\text {dummy }}^{\prime 1}$.

4. We next call Permute to the combined layout $\left\{\mathfrak{e},\left(\mathrm{U}_{b}, \mathrm{~L}_{b}\right)\right\}_{b \in \mathbb{Z}_{3}}$, where each server $\mathbf{S}_{b}$ has input $\pi_{b+1}$, to produce $\left\{\pi_{b},\left(\mathrm{U}_{b}^{\prime}, \mathrm{L}_{b}^{\prime}\right)\right\}_{b \in \mathbb{Z}_{3}}$ as output.

5. The linked lists can now be accessed using the four position tuples $\mathfrak{p}_{\text {real }}^{1}, \mathfrak{p}_{\text {real }}^{\prime 1}$, $\mathfrak{p}_{\text {dummy }}^{1}$, and $\mathfrak{p}_{\text {dummy }}^{\prime 1}$. The client first starts accessing real elements in the two lists using $\mathfrak{p}_{\text {real }}^{1}$ and $\mathfrak{p}_{\text {real }}^{1}$ to merge them. When a real list ends, it starts accessing the corresponding dummy list.

More precisely, the client secretly writes the merged result to the abstract output array $\mathrm{U}^{\prime \prime}$.

Start with $k \leftarrow 0, \mathfrak{p}^{1} \leftarrow \mathfrak{p}_{\text {real }}^{1}, \mathfrak{p}^{2} \leftarrow \mathfrak{p}_{\text {real }}^{2}$.

For each $s \in\{1,2\}$, the client reconstructs $\mathrm{B}^{s}:=\oplus_{b} \mathrm{U}_{b}^{\prime}\left[\mathfrak{p}_{b}^{s}\right]$ and next ${ }^{s}:=$ $\oplus_{b} \mathrm{~L}_{b}^{\prime}\left[\mathfrak{p}_{b}^{s}\right]$ at most once, i.e., if $\mathrm{B}^{s}$ and next ${ }^{s}$ have already been reconstructed once with the tuple $\left(\mathfrak{p}_{b}^{p}: b \in \mathbb{Z}_{3}\right)$, then they will not be reconstructed again. If $\mathrm{B}^{1}$ should appear before $\mathrm{B}^{2}$, then the client secretly writes $\mathrm{U}^{\prime \prime}[k] \leftarrow \mathrm{B}^{1}$ and updates $k \leftarrow k+1, \mathfrak{p}^{1} \leftarrow$ next $^{1}$; if the end of the real list is reached, then it updates $\mathfrak{p}^{1} \leftarrow \mathfrak{p}_{\text {dummy }}^{1}$. The case when $B^{2}$ should appear before $B^{1}$ is analogous.

The next element is processed until the client has secretly constructed the whole abstract array $\mathrm{U}^{\prime \prime}$.

6. The new merged layout $\left\{\mathfrak{e}, \mathrm{U}_{b}^{\prime \prime}\right\}_{b \in \mathbb{Z}_{3}}$ is produced.

Theorem 6. The Merge protocol perfectly securely realizes the ideal functionality $\mathcal{F}_{\text {merge }}$ in the presence of a semi-honest adversary corrupting a single server with $O(n)$ bandwidth. 
Proof. We follow the same strategy as in Theorem 5. Again, from the construction, the protocol performs merging correctly and re-distributes secretes using independent randomness. Hence, the marginal distribution of the outputs is the same for both the protocol and the ideal functionality.

We fix the inputs of all servers, and some $b \in \mathbb{Z}_{3}$. Recall that the goal is to show that (1) the view ${ }^{b}$ follows a distribution that is totally determined by the inputs $\mathbf{I}_{b}$ and the outputs $\mathbf{O}_{b}$ of $\mathbf{S}_{b} ;(2)$ conditioning on $\mathbf{O}_{b}$, view ${ }^{b}$ is independent of the outputs of the client and all other servers.

The second part is easy, because the inputs are fixed. Hence, conditioning on $\mathbf{O}_{b}$ (which includes $\mathrm{U}_{b}^{\prime \prime}$ and $\mathrm{U}_{b+1}^{\prime \prime}$ ), $\mathrm{U}_{b+2}^{\prime \prime}$ has no more randomness and totally determines the outputs of other servers.

To prove the first part, our strategy is to decompose view ${ }^{b}$ into a list components, and show that fixing $\mathbf{I}_{b}$ and conditioning on $\mathbf{O}_{b}$ and a prefix of the components, the distribution of the next component can be determined. Hence, this also gives the definition of a simulator.

First, observe that in the last step, the client re-distributes the shares of $\mathrm{U}^{\prime \prime}$, and gives output $\mathbf{O}_{b}$ (including $\mathrm{U}_{b}^{\prime \prime}$ and $\mathrm{U}_{b+1}^{\prime \prime}$ ) to $\mathbf{S}_{b}$; moreover, the shares of $\mathrm{U}^{\prime \prime}$ are generated with fresh independent randomness. Hence, the distribution of the part of view ${ }^{b}$ excluding $\mathbf{O}_{b}$ is independent of $\mathbf{O}_{b}$. We consider the components of view ${ }^{b}$ in the following order, which is also how a simulator generates a view after seeing $\mathbf{I}_{b}$.

1. Communication Pattern. Observe that from the description of the algorithm, the communication pattern between the client and the servers depends only on the length $n$ of the input arrays.

2. Random permutation $\pi_{b+1}$. This is independently generated using fresh randomness.

3. Link $\mathrm{L}$ creation. The (abstract) array $\mathrm{L}$ is created by reverse linear scan. The shares $\mathrm{L}_{b}$ and $\mathrm{L}_{b+1}$ received by $\mathbf{S}_{b}$ are generated by fresh independent randomness.

4. Permute subroutine. By the correctness of the Permute, the shares of the outputs $\left(\mathrm{U}^{\prime}, \mathrm{L}^{\prime}\right)$ received by $\mathbf{S}_{b}$ follow an independent and uniform random distribution. By the perfect security of Permute, the component of view ${ }^{b}$ due to Permute depends only on the inputs (which include the shares of $T$ and L) and the outputs of the subroutine.

5. List traversal. Since $\mathbf{S}_{b}$ does not know $\pi_{b}$ (which is generated using independent randomness by $\mathbf{S}_{b-1}$ ), from $\mathbf{S}_{b}$ 's point of view, while the elements from the two underlying lists are being merged, its array $\mathrm{U}_{b}^{\prime}$ is traversed in an independent and uniform random order.

Therefore, we have described a simulator procedure that samples the view ${ }^{b}$ step-by-step, given $\mathbf{I}_{b}$ and $\mathbf{O}_{b}$.

Efficiency. The analysis for the Merge protocol is similar to that for Theorem 5 except that the operations are performed on lists of size $2 n$ instead of $n$. 


\section{Three-Server One-Time Oblivious Memory}

We construct an abstract datatype to process non-recurrent memory lookup requests, i.e., between rebuilds of the data structure, each distinct address is requested at most once. Our abstraction is similar to the perfectly secure onetime oblivious memory by Chan et al. 5. However, while Chan et al. only consider perfect security with respect to access pattern, our three-server one time memory in addition information-theoretically encrypts the data itself. Thus, in [5], since the algorithm does not provide guarantees for the data itself, it can modify the data structure while performing operations. In contrast, our onetime oblivious memory is a read-only data structure. In this data structure, we assume every request is tagged with a position label indicating which memory location to lookup in each of the servers. In this section, we assume that such a position is magically available during lookup; but in subsequent sections we show how this data structure can be maintained and provided during a lookup.

\subsection{Definition: Three-server One-Time Oblivious Memory}

Our (three-server) one-time oblivious memory supports three operations: 1) Build, 2) Lookup, and 3) Getall. Build is called once upfront to create the data structure: it takes in a set of data blocks (tagged with its logical address), permutes shares of the data blocks at each of the servers to create a data structure that facilitates subsequent lookup from the servers. Once the data structure is built, lookup operations can be performed on it. Each lookup request consists of a logical address to lookup and a position label for each of the three servers, thereby enabling them to perform the lookup operation. The lookup can be performed for a real logical address, in which case the logical address and the position labels for each of the three servers are provided; or it can be a dummy request, in which case $\perp$ is provided. Finally, a Getall operation is called to obtain a list $U$ of all the blocks that were provided during the Build operation. Later, in our ORAM scheme, the elements in the list $U$ will be combined with those in other lists to construct a potentially larger one-time oblivious memory.

Our three-server one-time oblivious memory maintains obliviousness as long as 1) for each real block in the one-time memory, a lookup is performed at most once, 2) at most $n$ total lookups (all of which could potentially be dummy lookups) are performed, and 3) no two servers collude with each other to learn the shares of the other server.

Formal Definition. Our three-server one-time oblivious memory scheme OTM $[n]$ is parameterized by $n$, the number of memory lookup requests supported by the data structure. It is comprised of the following randomized, stateful algorithms:

$-\left(U,\left(\left\{\pi_{b},\left(\widehat{\mathrm{T}_{b}}, \widehat{\mathrm{L}_{b}}\right)\right\}_{b \in \mathbb{Z}_{3}}\right.\right.$, dpos $\left.)\right) \leftarrow \operatorname{Build}(\mathrm{T}, \perp):$

- Input: A sorted, unpermuted layout denoted $\left\{\mathfrak{e}, \mathrm{T}_{b}\right\}_{b \in \mathbb{Z}_{3}}$ representing an abstract sorted list T. T $[i]$ represents a key-value pair $\left(k_{i}, v_{i}\right)$ which are 
either real and contains a real address key ${ }_{i}$ and value $v_{i}$, or dummy and contains a $\perp$. The list T is sorted by the key key ${ }_{i}$. The client's input is $\perp$.

- Functionality: The Build algorithm creates a layout $\left\{\pi_{b},\left(\widehat{\mathrm{T}_{b}}, \widehat{\mathrm{L}_{b}}\right)\right\}_{b \in \mathbb{Z}_{3}}$ of size $2 n$ that will facilitate subsequent lookup requests; intuitively, $n$ extra dummy elements are added, and the $\widehat{\mathrm{L}_{b}}$ 's maintain a singly-linked list for these $n$ dummy elements. Moreover, the tuple of head positions is secretshared $\oplus_{b}$ dpos $_{b}$ among the three servers.

It also outputs a sorted list $U$ of $n$ key-value pairs (key, pos) sorted by key where each pos $:=\left(\operatorname{pos}_{0}, \operatorname{pos}_{1}, \operatorname{pos}_{2}\right)$; the invariant is that if key $\neq \perp$, then the data for key is $\oplus_{b} \widehat{T_{b}}\left[\operatorname{pos}_{b}\right]$.

The output list $U$ is stored as a sorted, unpermuted layout $\left\{\mathfrak{e}, U_{b}\right\}_{b \in \mathbb{Z}_{3}}$. Every real key from $T$ appears exactly once in $U$ and the remaining entries of $U$ are $\perp$ 's. The client's output is $\perp$.

Later in our scheme, $U$ will be propagated back to the corresponding data structure with preceding recursion depth during a coordinated rebuild. Hence, $U$ does not need to carry the value $v_{i}$ 's.

$-v \leftarrow \operatorname{Lookup}\left(\left(\left\{\pi_{b}, \widehat{\left(\mathrm{T}_{b}\right.}, \widehat{\mathrm{L}_{b}}\right)\right\}_{b \in \mathbb{Z}_{3}}\right.$, dpos $),($ key, pos $\left.)\right)$ :

- Input: The client provides a key key and a position label tuple pos := $\left(\operatorname{pos}_{0}, \operatorname{pos}_{1}, \operatorname{pos}_{2}\right)$. The servers input the data structure $\left\{\pi_{b},\left(\widehat{T_{b}}, \widehat{L_{b}}\right)\right\}_{b \in \mathbb{Z}_{3}}$ and dpos created during Build.

- Functionality: If key $\neq \perp$, return $\oplus_{b} \widehat{\widehat{T}_{b}}\left[\operatorname{pos}_{b}\right]$ else, return $\perp$.

$-R \leftarrow \operatorname{Getall}\left(\left\{\pi_{b},\left(\widehat{\mathrm{T}_{b}}, \widehat{\mathrm{L}}_{b}\right)\right\}_{b \in \mathbb{Z}_{3}}, \perp\right)$ :

- Input: The servers input the data structure $\left\{\pi_{b},\left(\widehat{\mathrm{T}_{b}}, \widehat{\mathrm{L}_{b}}\right)\right\}_{b \in \mathbb{Z}_{3}}$ created during Build.

- Functionality: the Getall algorithm returns a sorted, unpermuted layout $\left\{\mathfrak{e}, R_{b}\right\}_{b \in \mathbb{Z}_{3}}$ of length $n$. This layout represents an abstract sorted list $R$ of key-value pairs where each entry is either real and of the form (key, $v$ ) or dummy and of the form $(\perp, \perp)$. The list $R$ contains all real elements inserted during Build including those that have been looked up, padded with $(\perp, \perp)$ to a length of $n 7$

Valid request sequence. Our three-server one-time oblivious memory ensures obliviousness only if lookups are non-recurrent (i.e., the same real key is never looked up more than once); and the number of lookups is upper bounded by $n$, the size of the input list provided to Build. More formally, a sequence of operations is valid, iff the following holds:

- The sequence begins with a single call to Build, followed by a sequence of at most $n$ Lookup calls, and finally the sequence ends with a call to Getall.

- All real keys in the input provided to Build have distinct keys.

7 The Getall function returns as output the unpermuted layout that was input to Build. It primarily exists for ease of exposition. 
- For every Lookup concerning a real element with client's input (key, pos := $\left.\left(\operatorname{pos}_{0}, \operatorname{pos}_{1}, \operatorname{pos}_{2}\right)\right)$, the key should have existed in the input to Build. Moreover, the position label tuple $\left(\operatorname{pos}_{0}, \operatorname{pos}_{1}, \operatorname{pos}_{2}\right)$ must be the correct position labels for each of the three servers.

- No two Lookup requests should request the same real key.

Correctness. Correctness requires that:

1. For any valid request sequence, with probability 1 , every Lookup request must return the correct value $v$ associated with key key that was supplied in the Build operation.

2. For any valid request sequence, with probability 1 , Getall must return an array $R$ containing every (key, $v$ ) pair that was supplied to Build, padded with dummies to have $n$ entries.

Perfect obliviousness. Suppose the following sequence of operations are executed: the initial Build, followed by a valid request sequence of $\ell$ Lookup's, and the final Getall. Perfect obliviousness requires that for each $b \in \mathbb{Z}_{3}$, the joint distribution of the communication pattern (between the client and the servers) and the view ${ }^{b}$ of $\mathbf{S}_{b}$ is fully determined by the parameters $n$ and $\ell$.

\subsection{Construction}

Intuition. The intuition is to store shares of the input list on storage servers such that each share is independently permuted and each server storing a share does not know its permutation (but some other server does). In order to lookup a real element, if a position label for all three shares are provided, then the client can directly access the shares. Since the shares are permuted and the server storing a share does not know the permutation, each lookup corresponds to accessing a completely random location and is thus perfectly oblivious. This is true so far as each element is accessed exactly once and the position label provided is correct; both of these constraints are satisfied by a valid request sequence. However, in an actual request sequence, some of the requests may be dummy and these requests do not carry a position label with them. To accommodate dummy requests, before permuting the shares, we first append shares of dummy elements to shares of the unpermuted input list. We add dummy elements enough to support all lookup requests before the one time memory is destroyed. Then we create a linked list of dummy elements so that a dummy element stores the position label of the location where the next dummy element is destined to be after permutation. The client maintains the head of this linked list, updating it every time a dummy request is made. To ensure obliviousness, the links (position labels) in the dummy linked list are also stored secret-shared and permuted along with the input list.

Protocol Build. Our oblivious Build algorithm proceeds as follows. Note that the input list $T$ is stored as an unpermuted layout $\left\{\mathfrak{e}, T_{b}\right\}_{b \in \mathbb{Z}_{3}}$ on the three servers. 
1. Initialize to add dummies. Construct an extended abstract $\mathrm{T}^{\prime}[0 . .2 n-1]$ of length $2 n$ such that the first $n$ entries are key-value pairs copied from the input $\mathrm{T}$ (some of which may be dummies).

The last $n$ entries of $\mathrm{T}^{\prime}$ contain special dummy keys. For each $i \in[1 . . n]$, the special dummy key $i$ is stored in $\mathrm{T}^{\prime}[n-1+i]$, and the entry has a keyvalue pair denoted by $\perp_{i}$. For each $i \in[1 . . n]$, the client secretly writes $\perp_{i}$ to $\mathbf{T}^{\prime}[n-1+i]$.

2. Generate permutations for OTM. Each server $\mathbf{S}_{b}$ acts as the permutation server for $\mathbf{S}_{b+1}$. For each $b \in \mathbb{Z}_{3}$, the client generates a random permutation $\pi_{b+1}:[2 n] \rightarrow[2 n]$ on permutation server $\mathbf{S}_{b}$.

3. Construct a dummy linked list. Using the newly generated permutation $\pi_{b+1}$ on server $\mathbf{S}_{b}$, the client constructs a linked list of dummy blocks. This is to enable accessing the dummy blocks linearly, i.e., for each $i \in[1 . . n-1]$, after accessing dummy block $\perp_{i}$, the client should be able to access $\perp_{i+1}$.

The client simply leverages $\pi_{b+1}(n . .2 n-1)$ stored on server $\mathbf{S}_{b}$ to achieve this. Specifically, for $i$ from $n-1$ down to 1 , to create a link between $i$-th and $(i+1)$-st dummy, the client reads $\pi_{b+1}(n+i)$ from server $\mathbf{S}_{b}$ and secretly writes the tuple $\left(\pi_{b+1}(n+i): b \in \mathbb{Z}_{3}\right)$ to the abstract link $\mathrm{L}[n+i-1]$.

There are no links between real elements, i.e., for $j \in[0 . . n-1]$, the client secretly writes $(\perp, \perp, \perp)$ to (abstract) $\mathrm{L}[j]$.

Observe that these links are secret-shared and stored as an unpermuted layout $\left\{\mathfrak{e}, \mathrm{L}_{b}\right\}_{b \in \mathbf{S}_{b}}$.

Finally, the client records the positions of the head of the lists and secretly writes the tuple across the three servers, i.e., $\oplus_{b}$ dpos $_{b}:=\left(\pi_{b}(n): b \in \mathbb{Z}_{3}\right)$, where $\operatorname{dpos}_{b}$ is stored on server $\mathbf{S}_{b}$.

4. Construct the key-position map $U$. The client can construct the (abstract) key-position map $U[0 . . n-1]$ sorted by the key from the first $n$ entries of $\mathrm{T}^{\prime}$ and the $\pi_{b}$ 's. Specifically, for each $i \in[0 . . n-1]$, the client secretly writes $\left(\operatorname{key}_{i},\left(\pi_{b}(i): b \in \mathbb{Z}_{3}\right)\right)$ to $U[i]$.

Recall that $U$ is stored as a sorted, unpermuted layout $\left\{\mathfrak{e}, U_{b}\right\}_{b \in \mathbb{Z}_{3}}$.

5. Permute the lists along with the links. Invoke Permute with input $\left\{\mathfrak{e},\left(\mathrm{T}_{b}^{\prime}, \mathrm{L}_{b}\right)\right\}_{b \in \mathbb{Z}_{3}}$, and permutation $\pi_{b+1}$ as the input for $\mathbf{S}_{b}$. The Permute protocol returns a

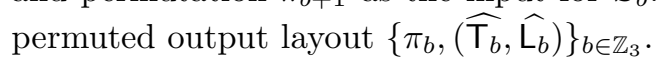

6. As the data structure, each server $\mathbf{S}_{b}$ stores $\left(\widehat{\mathrm{T}_{b}}, \widehat{\mathrm{L}_{b}}\right),\left(\pi_{b+1},\left(\widehat{\mathrm{T}_{b+1}}, \widehat{\mathrm{L}_{b+1}}\right)\right)$, and dpos $_{\mathrm{b}+1}$. The algorithm returns key-position map list $U$ as output, which is stored as an unpermuted layout $\left\{\mathfrak{e}, U_{b}\right\}_{b \in \mathbb{Z}_{3}}$. This list will later be passed to the preceding recursion depth in the ORAM scheme during a coordinated rebuild operation.

Fact 7 The Build algorithm for building an OTM supporting $n$ lookups requires an $O(n)$ bandwidth.

Proof. Each of the steps in the protocol either generate a random permutation using Fisher-Yates, invoke Permute, or linearly scan lists of size $O(n)$ blocks. Each of these steps can be performed with an $O(n)$ bandwidth. 
Protocol Lookup. Our oblivious Lookup $\left(\left(\left\{\pi_{b},\left(\widehat{T_{b}}, \widehat{L_{b}}\right)\right\}_{b \in \mathbb{Z}_{3}}\right.\right.$, dpos $),\left(\right.$ key, $\left.\left.\left(\operatorname{pos}_{0}, \operatorname{pos}_{1}, \operatorname{pos}_{2}\right)\right)\right)$ algorithm proceeds as follows:

1. The client reconstructs $\left(\operatorname{pos}_{0}^{\prime}, \operatorname{pos}_{1}^{\prime}, \operatorname{pos}_{2}^{\prime}\right) \leftarrow \oplus_{b}$ dpos $_{b}$.

2. Decide position to fetch from. If key $\neq \perp$, set $\operatorname{pos} \leftarrow\left(\operatorname{pos}_{0}, \operatorname{pos}_{1}, \operatorname{pos}_{2}\right)$, i.e., we want to use the position map supplied from the input; if key $=\perp$, set pos $\leftarrow\left(\operatorname{pos}_{0}^{\prime}, \operatorname{pos}_{1}^{\prime}, \operatorname{pos}_{2}^{\prime}\right)$, i.e., the dummy list will be used.

3. Reconstruct data block. Reconstruct $v \leftarrow \oplus \widehat{\mathrm{T}_{b}}\left[\operatorname{pos}_{b}\right]$ and $\left(\widehat{\operatorname{pos}_{0}}, \widehat{\operatorname{pos}_{1}}, \widehat{\operatorname{pos}_{2}}\right) \leftarrow$ $\oplus \widehat{\mathrm{L}_{b}}\left[\operatorname{pos}_{b}\right]$.

4. Update head of the dummy linked list. If key $\neq \perp$, the client re-shares the secrets $\oplus_{b}$ dpos $_{b} \leftarrow\left(\operatorname{pos}_{0}^{\prime}\right.$, $\left.\operatorname{pos}_{1}^{\prime}, \operatorname{pos}_{2}^{\prime}\right)$ with the same head; if key $=\perp$, the client secretly shares the updated head $\oplus_{b} \operatorname{dpos}_{b} \leftarrow\left(\widehat{\operatorname{pos}}_{0}, \widehat{\operatorname{pos}}_{1}, \widehat{\operatorname{pos}}_{2}\right)$.

5. Read value and return. Return $v$.

Fact 8 The Lookup algorithm for looking up a block in OTM requires $O(1)$ bandwidth.

Protocol Getall. For Getall, the client simply invokes the Unpermute protocol on input layout $\left.\left\{\pi_{b}, \widehat{\left(\bar{T}_{b}\right.}, \widehat{L_{b}}\right)\right\}_{b \in \mathbb{Z}_{3}}$ and returns the first $n$ entries of the sorted, unpermuted layout (and ignores the links created). This output is also stored as a sorted, unpermuted layout $\left\{\mathfrak{e}, \mathrm{T}_{b}\right\}_{b \in \mathbb{Z}_{3}}$. The data structure created on the servers during Build can now be destroyed.

Fact 9 The OTM Getall algorithm requires an $O(n)$ bandwidth.

Lemma 1. The subroutines Build, Lookup and Getall are correct and perfectly oblivious in the presence of a semi-honest adversary corrupting a single server.

Proof. We go through each subroutine and explain its purpose, from which correctness follows; moreover, we also argue why it is perfectly oblivious. Fix some $b \in \mathbb{Z}_{3}$, and we consider the view ${ }^{b}$ of corrupt $\mathbf{S}_{b}$.

Build subroutine. We explain each step, and why the corresponding view ${ }^{b}$ satisfies perfect obliviousness.

1. Initialize to add dummies. This step appends $n$ dummies at the end of the abstract array. The data access pattern depends only $n$. The contents of the extra dummy entries are secretly shared among the servers using independent randomness.

2. Generate permutations for OTM. The client generates an independent random permutation for each server. In particular, the permutation $\pi_{b+1}$ received by $\mathbf{S}_{b}$ is independent and uniformly random.

3. Construct a dummy linked list. In this step, a linked list is created for the dummy entries. Using $\pi_{b+1}$, server $\mathbf{S}_{b}$ observes a linear scan on its array and creates a linked list that is meant for $\mathbf{S}_{b+1}$. Similarly, the linked list for $\mathbf{S}_{b}$ is created by $\mathbf{S}_{b-1}$.

The links themselves are secretly shared and stored in the abstract $\mathrm{L}$, and so are the head positions $\oplus_{b} \mathrm{dpos}_{b}$. Hence, the data seen by $\mathbf{S}_{b}$ appears like independent randomness. 
4. Construct the key-position map $U$. This step is just a linear scan on the abstract $T^{\prime}$ and each permutation array $\pi_{b}$. The resulting abstract array $U$ is also secretly shared.

5. Permute the lists along with the links. This steps uses the building block Permute, which is proved to be perfectly secure in Lemma 3 ,

6. Finally, as guaranteed by Permute, the resulting arrays $\widehat{T}$ and $\hat{L}$ are secretly shared using independent randomness. Moreover, the abstract list $U$ is returned as required. Hence, the Build subroutine is correct and perfectly oblivious, as required.

Lookup Subroutine. By construction, each call to Lookup is supplied with the correct position labels, and hence, correctness is achieved. We next argue why the distribution of the view ${ }^{b}$ of corrupt $\mathbf{S}_{b}$ depends only on the number $\ell$ of lookups.

Observe that the entries accessed in $\mathbf{S}_{b}$ are permuted randomly by $\pi_{b}$, which is unknown to $\mathbf{S}_{b}$. Since the request sequence is non-recurrent, each real key is requested at most once. On the other hand, the dummy entries are singly linked, and each dummy entry is accessed also at most once. Hence, the $\ell$ lookups correspond to $\ell$ distinct uniformly random accesses in $\mathbf{S}_{b}$ 's corresponding arrays. Getall Subroutine. This subroutine calls the building block Unpermute, which is proved to be perfectly secure in Lemma 4 .

As mentioned before, all data stored on $\mathbf{S}_{b}$ is secretly shared using independent randomness. Hence, the distribution of the overall view ${ }^{b}$ depends only on the number $\ell$ of lookups.

\section{$5 \quad 3$-Server ORAM with $O\left(\log ^{2} N\right)$ Simulation Overhead}

\subsection{Position-Based ORAM}

Our ORAM scheme will consist of logarithmically many position-based ORAMs of geometrically increasing sizes, henceforth denoted ORAM $\mathrm{ORAM}_{1}, \ldots, \mathrm{ORAM}_{D}$ where $D:=\log _{2} N$. See Figure 1 for a high level sketch of our construction.

Specifically, ORAM ${ }_{d}$ stores $\Theta\left(2^{d}\right)$ blocks where $d \in\{0,1, \ldots, D\}$. The actual data blocks are stored in $\mathrm{ORAM}_{D}$ whereas all other $\mathrm{ORAM}_{d}, d<D$ recursively stores position labels for the next depth $d+1$.

In this subsection, we focus on describing ORAM $_{d}$ assuming the position labels are magically available. In the next subsection, we will describe how position labels are maintained across different depths.

Data Structure. For $0 \leq d \leq D$ each ORAM $_{d}$ consists of $d+1$ levels of three-server one-time oblivious memory that are geometrically increasing in size. We denote these one-time oblivious memories as $\left(\mathrm{OTM}_{j}: j=0, \ldots, d\right)$ where $\mathrm{OTM}_{j}:=\mathrm{OTM}\left[2^{j}\right]$ stores at most $2^{j}$ real blocks.

Every level $j$ is marked as either empty (when the corresponding OTM $_{j}$ has not been built) or full (when OTM ${ }_{j}$ is ready and in operation). Initially, all levels are empty. 


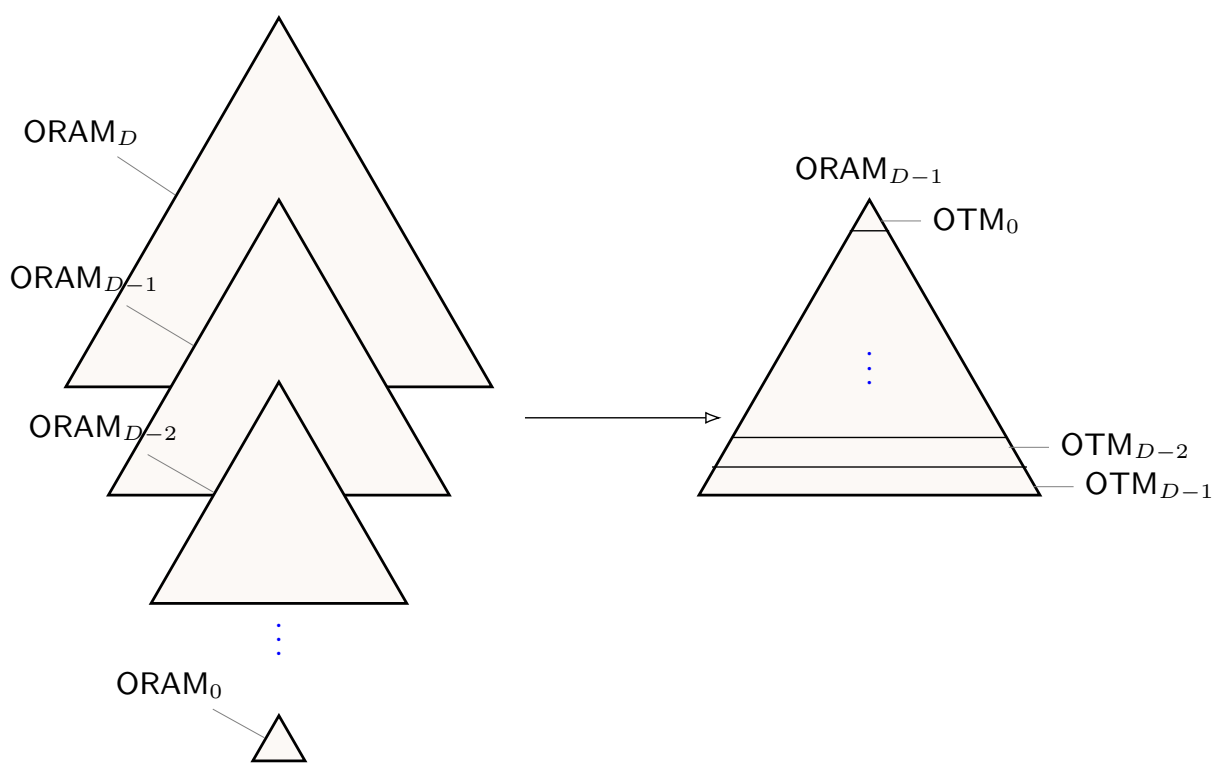

Fig. 1: ORAM Construction from Position-Based ORAM

Position label. To access a block stored in $\mathrm{ORAM}_{d}$, its position label specifies 1) the level $l \in[0 . . d]$ such that the block resides in OTM $\ell$; and 2) the tuple pos $:=\left(\operatorname{pos}_{0}, \operatorname{pos}_{1}, \operatorname{pos}_{2}\right)$ to reconstruct the block from OTM .

Operations Each position-based ORAM supports two operations, Lookup and Shuffle.

Protocol Lookup:

- Input: The client provides (key, pos $\left.:=\left(l,\left(\operatorname{pos}_{0}, \operatorname{pos}_{1}, \operatorname{pos}_{2}\right)\right)\right)$ as input, where key is the logical address for the lookup request, $l$ represents the level such that the block is stored in OTM ${ }_{l}$, and $\left(\operatorname{pos}_{0}, \operatorname{pos}_{1}, \operatorname{pos}_{2}\right)$ is used as an argument for $\mathrm{OTM}_{l}$.Lookup.

The servers store OTM $_{j}$ for $0 \leq j \leq d$ where OTM stores layout $\left\{\pi_{b},\left(\widehat{T_{b}}, \widehat{L_{b}}\right)\right\}_{b \in \mathbb{Z}_{3}}$ and dpos for the level. Moreover, some of the OTMs may be empty.

- Algorithm: The lookup operation proceeds as follows:

1. For each non-empty level $j=0, \ldots, d$, perform the following:

- The position label specifies that the block is stored at level OTM $\mathrm{OT}_{l}$. For level $j=l$, set key' $:=$ key and $\operatorname{pos}^{\prime}:=\left(\operatorname{pos}_{0}, \operatorname{pos}_{1}, \operatorname{pos}_{2}\right)$. For all other levels, set key' $:=\perp, \operatorname{pos}^{\prime}:=\perp$.

- $v_{j} \leftarrow \operatorname{OTM}_{j} \cdot \operatorname{Lookup}\left(\left(\left\{\pi_{b},\left(\widehat{\mathrm{T}_{b}}, \widehat{\mathrm{L}_{b}}\right)\right\}_{b \in \mathbb{Z}_{3}}, \mathrm{dpos}\right),\left(\right.\right.$ key $^{\prime}$, pos $\left.\left.^{\prime}\right)\right)$.

2. Return $v_{l}$.

Fact 10 For ORAM $_{d}$, Lookup requires an $O(d)$ bandwidth. 
Protocol Shuffle. The shuffle operation is used in hierarchical ORAMs to shuffle data blocks in consecutive smaller levels and place them in the first empty level (or the largest level). Our shuffle operation, in addition, accepts another input $U$ that is used to update the contents of data blocks stored in the position based ORAM. In the final ORAM scheme, the list $U$ passed as an input to ORAM $_{d}$ will contain the (new) position labels of blocks in $\mathrm{ORAM}_{d+1}$. Similarly, the shuffle operation returns an output $U^{\prime}$ that will be passed as input to ORAM $_{d-1}$. More formally, our shuffle operation can be specified as follows:

$\left(U^{\prime}, \widehat{T}\right) \leftarrow$ Shuffle $_{d}\left(\left(\mathrm{OTM}_{0}, \ldots, \mathrm{OTM}_{l}, U\right), l\right):$

- Input: The shuffle operation for $\mathrm{ORAM}_{d}$ accepts as input from the client a level $l$ in order to build $\mathrm{OTM}_{l}$ from data blocks currently in levels $0, \ldots, l$. In addition, ORAM $_{d}$ consists of an extra OTM, denoted by OTM $_{0}^{\prime}$, containing only a single element. Jumping ahead, this single element represents a freshly fetched block.

The inputs of the servers consist of OTMs for levels up to level $l$, each of which is stored as a permuted layout $\left\{\pi_{b},\left(\widehat{T_{b}}, \widehat{L_{b}}\right)\right\}_{b \in \mathbb{Z}_{3}}$ and an array of keyvalue pairs $U$, stored as a sorted, unpermuted layout $\left\{\mathfrak{e}, U_{b}\right\}_{b \in \mathbb{Z}_{3}}$. The array $U$ is used to update the blocks during the shuffle operation.

Throughout the shuffle operation we maintain the following invariant:

- For every $\mathrm{ORAM}_{d}, l \leq d$. Moreover, either level $l$ is the smallest empty level of $\mathrm{ORAM}_{d}$ or $l$ is the largest level, i.e., $l=d$.

- Each logical address appears at most once in $U$.

- The input $U$ contains a subset of logical addresses that appear in levels $0, \ldots, l$ of the $\mathrm{ORAM}_{d}\left(\right.$ or $\left.\mathrm{OTM}_{0}^{\prime}\right)$.

Specifically, given a key-value pair (key, $v$ ), the corresponding block (key, $v^{\prime}$ ) should already appear in some level in $[0 . . l]$ or OTM $\mathrm{O}_{0}^{\prime}$. An update rule will determine how $v$ and $v^{\prime}$ are combined to produce a new value $\widehat{v}$ for key.

- The Shuffle algorithm proceeds as follows:

1. Retrieve key-value pairs from $\left(\mathrm{OTM}_{0}, \ldots, \mathrm{OTM}_{l}\right)$. The client first retrieves the key-value pairs of real blocks from $\left(\mathrm{OTM}_{0}, \ldots, \mathrm{OTM}_{l}\right)$ and restore each array to its unpermuted form. More specifically, the client constructs the unpermuted sorted $\mathrm{T}^{j} \leftarrow \mathrm{OTM}_{j}$. Getall $\left(\left\{\pi_{b},\left(\widehat{\mathrm{T}}_{b}, \widehat{\mathrm{L}}_{b}\right)\right\}_{b \in \mathbb{Z}_{3}}, \perp\right)$, for $0 \leq j \leq l$, and $\mathrm{T}^{0} \leftarrow \mathrm{OTM}_{0}^{\prime}$. Getall $\left(\left\{\pi_{b},\left(\widehat{\mathrm{T}_{b}}, \widehat{\mathrm{L}_{b}}\right)\right\}_{b \in \mathbb{Z}_{3}}, \perp\right) 8$ Now, the old $\mathrm{OTM}_{0}, \ldots, \mathrm{OTM}_{l}$ instances can be destroyed.

2. Create a list for level $l$. The client then creates a level $l$ list of keys from $\left(\mathrm{OTM}_{0}, \ldots, \mathrm{OTM}_{l}\right)$.

- Merge lists from consecutive levels to form level l list. The merge procedure proceeds as follows:

For $j=0, \ldots, l-1$ do: $\widehat{\mathrm{T}}^{j+1} \leftarrow \operatorname{Merge}\left(\left(\widehat{\mathrm{T}}^{j}, \mathrm{~T}^{j}\right), \perp\right)$ where $\mathrm{T}^{j}$ and $\widehat{\mathrm{T}}^{j}$ are of size $2^{j}$

Moreover, the lists are individually sorted but may contain blocks that have already been accessed. In the Merge protocol, for two elements with

\footnotetext{
${ }^{8}$ The layout inputs to the Getall operation are restricted to the ones stored in $\mathrm{OTM}_{j}$ for $0 \leq j \leq l$, respectively.
} 
the same key and belonging to different OTM levels, we prefer the one at the smaller level first. For the case where $l=d$, perform another merge $\widehat{\mathrm{T}}^{d} \leftarrow \operatorname{Merge}\left(\left(\widehat{\mathrm{T}}^{d}, \mathrm{~T}^{d}\right), \perp\right)$ to produce an array of size $2^{d+1}$; Jumping ahead, the size will be reduced back to $2^{d}$ in subsequent steps.

At the end of this step, we obtain a merged sorted list $\widehat{\mathrm{T}}^{l}$, stored as $\widehat{\mathrm{T}}^{l}:=\left\{\mathfrak{e}, \widehat{\mathrm{T}}_{b}^{l}\right\}_{b \in \mathbb{Z}_{3}}$, containing duplicate keys that are stored multiple times (with potentially different values).

- Mark duplicate keys as dummy. From the stored duplicate keys, we only need the value of the one that corresponds to the latest access. All other duplicate entries can be marked as dummies. At a high level, this can be performed in a single pass by the client by scanning consecutive elements of the unpermuted sorted layout $\widehat{\mathrm{T}}^{l}$. The client keeps the most recent version, i.e., the version that appears first (and has come from the smallest OTM), and marks other versions as dummies. To maintain obliviousness, the secret shares need to be re-distributed for each scanned entry.

More specifically, suppose that there are $\lambda$ duplicate keys. Then, the client scans through the unpermuted layout $\widehat{T}^{l}:=\left\{\mathfrak{e}, \widehat{T}_{b}^{l}\right\}_{b \in \mathbb{Z}_{3}}$. For consecutive $\lambda$ elements, $j, \ldots, j+\lambda-1$ with the same key, the client re-distributes the secret for $\widehat{\mathrm{T}}^{l}[j]$ for position $j$, and secretly writes $\perp$ for positions $j+1, \ldots, j+\lambda-1$.

After this step, the resulting (abstract) $\widehat{T}^{l}$ is semi-sorted.

- Compaction to remove dummies. The client invokes the StableCompact protocol with input $\widehat{\mathrm{T}}^{l}:=\left\{\mathfrak{e}, \widehat{\mathrm{T}}_{b}^{l}\right\}_{b \in \mathbb{Z}_{3}}$, i.e., $\widehat{\mathrm{T}}^{l} \leftarrow \operatorname{StableCompact}\left(\widehat{\mathrm{T}}^{l}, \perp\right)$ to obtain a sorted, unpermuted layout (where the dummies are at the end). We keep the first $2^{l}$ entries.

3. Update $\widehat{\mathrm{T}}^{l}$ with values from $U$. The client updates $\widehat{\mathrm{T}}^{l}$ so that it contains updated position values from $U$. Looking ahead, in our final scheme, $U$ will contain the new position labels from an ORAM at a larger depth. Given that $\mathrm{ORAM}_{D}$ is the largest depth and does not store position values, this step is skipped for $\mathrm{ORAM}_{D}$.

We do this as follows:

- Merge $\widehat{\mathrm{T}}^{l}$ with $U$. The client performs $A \leftarrow \operatorname{Merge}\left(\left(\widehat{\mathrm{T}}^{l}, U\right), \perp\right)$ to obtain a sorted, unpermuted layout. Ties on the same key break by choosing the blocks in $\mathrm{T}^{l}$.

- Scan and Update A. In a single pass through the sorted, unpermuted layout $A$, it can operate on every adjacent pair of entries. If they share the same key, the following update rule is used to update both the values (the precise update rule is provided in the Convert subroutine in Section 5.2). In particular, in the final ORAM scheme, the keys in $A$ correspond to logical addresses. Each address in a position-based ORAM at depth- $d$ stores position labels for two children addresses at depth- $(d+1)$. The entries in $A$ that come from $\widehat{\mathrm{T}}^{l}$ contain the old position labels for both children. For the entries from $U$, if children position labels exist, they correspond to the new labels. For each of the child addresses, if $U$ contains a new position label, the update function chooses the new one; otherwise, it chooses the old label from $\widehat{T}^{l}$. 
- Compaction to remove dummies. The client invokes the StableCompact protocol $A \leftarrow$ StableCompact $(A, \perp)$ to obtain an updated sorted, unpermuted layout $A$. We keep the first $2^{l}$ entries.

4. Build $\mathrm{OTM}_{l}$. The client invokes $U^{\prime} \leftarrow \operatorname{Build}(A, \perp)$ to generate a data structure OTM $\mathrm{O}_{l}$ and $U^{\prime}$. Mark OTM $\mathrm{OT}_{l}$ as full and OTM $\mathrm{O}_{i}$, for $i<l$, as empty.

We now prove that the above position-based ORAM is correct and satisfies perfect obliviousness in the presence of a semi-honest adversary corrupting a single server. We also prove that an $\mathrm{ORAM}_{d}$, and $l \leq d$, Shuffle ${ }_{d}$ for level $l$ requires a bandwidth of $O\left(2^{l}\right)$. We use the same proof framework as [5]. However, instead of having only one server, we argue perfect obliviousness from an adversary that has access to only one of the three servers.

Fact 11 (Correctness) Our Position-based ORAM maintains correctness. More specifically, at every recursion depth $d$, the correct position labels will be input to the Lookup operations of $\mathrm{ORAM}_{d}$; and every request will return the correct answers.

Proof. Straightforward by construction.

For every $\mathrm{ORAM}_{d}$ at recursion depth $d$, the following invariant is respected by construction as stated in the following facts.

Fact 12 (Lifetime of OTM) For every $\mathrm{ORAM}_{d}$, every $\mathrm{OTM}_{i}$ instance at level $i \leq d$ that is created needs to answer at most $2^{i}$ requests before $\mathrm{OTM}_{i}$ instance is destroyed.

Proof. For every $\mathrm{ORAM}_{d}$, the following is true: imagine that there is a $(d+1)$-bit binary counter initialized to 0 that increments whenever a request comes in. Now, for $0 \leq \ell<d$, whenever the $\ell$-th bit flips from 1 to 0 , the $\ell$-th level of OTM is destroyed; whenever the $\ell$-th bit flips from 0 to 1 , the $\ell$-th level of OTM $\mathrm{OT}_{\ell}$ is reconstructed. For the largest level $d$ of $\mathrm{ORAM}_{d}$, whenever the $d$-th (most significant) bit of this binary counter flips from 0 to 1 or from 1 to 0 , the $(d+1)$ st level is destroyed and reconstructed. The fact follows in a straightforward manner by observing this binary-counter argument.

Fact 13 For every $\mathrm{ORAM}_{d}$ and every $\mathrm{OTM}_{\ell}$ instance at level $\ell \leq d$, during the lifetime of the OTM $\mathrm{OM}_{\ell}$ intance: (a) no two real requests will ask for the same key; and (b) for every request that asks for a real key, a block with key must exist in $\mathrm{OTM}_{i}$.

Proof. We first prove claim (a). Observe that for any $\mathrm{ORAM}_{d}$, if the block with some key is fetched from some level $\ell \leq d$, at this moment, that block will either enter a smaller level $\ell^{\prime}<\ell$; or some level $\ell^{\prime \prime} \geq \ell$ will be rebuilt and the block with key will go into level $\ell^{\prime \prime}$ - in the latter case, level $\ell$ will be destroyed prior to the rebuilding of level $\ell^{\prime \prime}$. In either of the above cases, due to correctness of the construction, if the key is needed again from $\mathrm{ORAM}_{d}$, a correct position label will be provided for key such that the request will not go to level $\ell$ (until the level is reconstructed). Finally, claim (b) follows from correctness of the position labels. 
Given the above facts, our construction maintains perfect obliviousness as in 5. We emphasize that while the proof structure is the same, our notion of perfect obliviousness is defined in terms of the view of one server plus the communication pattern between the client and all the servers.

Lemma 2 (Obliviousness). The above position-based ORAM construction satisfies perfect obliviousness in the presence of a semi-honest adversary corrupting a single server.

Proof. We would like to point out the major difference from [5]. Since we do not rely on cryptographic assumptions, the data content is protected by secretsharing among the three servers.

For every OTM instance constructed during the lifetime of the ORAM, Facts 12 and 13 are satisfied, and thus every one-time memory instance receives a valid request sequence. The lemma then follows in a straightforward fashion by the perfect obliviousness of the OTM scheme (and the perfect security of the underlying building blocks such as Merge), and by observing that all other access patterns of the ORAM construction are deterministic and independent of the input requests.

Fact 14 Suppose that the update function can be evaluated in $O(1)$ time. Then, for an $\mathrm{ORAM}_{d}$, and $l \leq d$, Shuffle ${ }_{d}$ for level $l$ requires a bandwidth of $O\left(2^{l}\right)$.

Proof. Observe that to obtain real key-value pairs from OTM's and to construct a level $l$ list, the client invokes Getall and Merge protocols on (abstract) lists of sizes $2^{0}, \ldots, 2^{l}$, and StableCompact on size $O\left(2^{l}\right)$. Since each of these steps require linear number of block operations, the total number of block operations for these steps is $\sum_{i=1}^{l}\left(2^{i}\right)+O\left(2^{l}\right)=O\left(2^{l}\right)$. In order to update the list to contain values from $U$, we again invoke Merge, StableCompact, and an update on a list of size $O\left(2^{l}\right)$. This requires $O\left(2^{l}\right)$ block operations assuming the update rule itself requires $O(1)$ operations per block. Finally, the call to Build $\mathrm{OTM}_{l}$ requires $O\left(2^{l}\right)$ block operations.

\subsection{ORAM Construction from Position-Based ORAM}

Our ORAM scheme consists of $D+1$ position-based ORAMs denoted as ORAM , $\ldots$, ORAM $_{D}$ where $D=\log _{2} N$. ORAM ${ }_{D}$ stores data blocks whereas ORAM for $d<D$ stores a position map for ORAM OR+1 $_{d}$. The previous section specified the construction of a position-based ORAM. However, it assumed that position labels are magically available at some ORAM ${ }_{d}$. In this section, we show a full ORAM scheme and specify 1 ) how these position labels for ORAM Ore obtained $_{d}$ from ORAM ${ }_{d-1}$, and 2) after a level of $\mathrm{ORAM}_{d}$ is built, how the position labels of blocks from the new level are updated at $\mathrm{ORAM}_{d-1}$.

Format of block address at depth d. Suppose that a block's logical address is a $\log _{2} N$-bit string denoted by $\operatorname{addr}^{\langle D\rangle}:=\operatorname{addr}\left[1 . .\left(\log _{2} N\right)\right]$ (expressed in binary format), where $\operatorname{addr}[1]$ is the most significant bit. In general, at depth $d$, an 
address addr ${ }^{\langle d\rangle}$ is the length- $d$ prefix of the full address addr ${ }^{\langle D\rangle}$. Henceforth, we refer to $\operatorname{addr}^{\langle d\rangle}$ as a depth- $d$ address (or the depth- $d$ truncation of addr).

When we look up a data block, we would look up the full address addr ${ }^{\langle D\rangle}$ in recursion depth $D$; we look up addr ${ }^{\langle D-1\rangle}$ at depth $D-1$, $\operatorname{addr}^{\langle D-2\rangle}$ at depth $D-2$, and so on. Finally at depth 0 , only one block is stored at ORAM . $_{0}$

A block with the address addr ${ }^{\langle d\rangle}$ in $\mathrm{ORAM}_{d}$ stores the position labels for two blocks in $\mathrm{ORAM}_{d+1}$, at addresses addr ${ }^{\langle d\rangle} \| 0$ and $\operatorname{addr}^{\langle d\rangle} \| 1$ respectively. Henceforth, we say that the two addresses addr ${ }^{\langle d\rangle} \| 0$ and $\operatorname{addr}^{\langle d\rangle} \| 1$ are siblings to each other; $\operatorname{addr}^{\langle d\rangle}|| 0$ is called the left sibling and $\operatorname{addr}^{\langle d\rangle} \| 1$ is called the right sibling. We say that $\operatorname{addr}^{\langle d\rangle} \| 0$ is the left child of $\operatorname{addr}^{\langle d\rangle}$ and $\operatorname{addr}^{\langle d\rangle} \| 1$ is the right child of $\operatorname{addr}^{\langle d\rangle}$.

An ORAM Lookup An ORAM lookup request is denoted as (op, addr, data) where op $\in\{$ read, write $\}$. If op $=$ read then data $:=\perp$. Here, addr denotes the address to lookup from the ORAM. The inputs are all provided by the client whereas the servers store position-based $\mathrm{ORAM}_{0}, \ldots, \mathrm{ORAM}_{D}$ as discussed in the previous section. We perform the following operations:

1. Fetch. For $d:=0$ to $D$, perform the following:

- Let addr ${ }^{\langle d\rangle}$ denote the depth- $d$ truncation of addr ${ }^{\langle D\rangle}$.

- Call ORAM OLookup to lookup addr $^{\langle d\rangle}$. Recall that the position labels for the

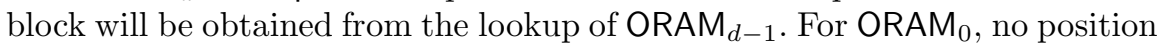
label is needed.

- The block returned from Lookup is placed in a special OTM $_{0}^{\prime}$ in ORAM O $_{\text {. }}$ Jumping ahead, this will be merged with the rest of the data structure in the maintain phase.

- If $d<D$, each lookup will return two positions for addresses addr ${ }^{\langle d\rangle} \| 0$ and $\operatorname{addr}^{\langle d\rangle} \| 1$. One of these will correspond to the position of $\operatorname{addr}^{\langle d+1\rangle}$ which will be required in the lookup for ORAM OR+1 $_{\text {. }}$.

- If $d=D$, the outcome of Lookup will contain the data block fetched.

2. Maintain. We first consider depth $D$. Set depth- $D$ 's update array $U^{D}:=\emptyset$. Suppose $l^{D}$ is the smallest empty level in $\mathrm{ORAM}_{D}$. We have the invariant that for all $0 \leq d<D$, if $l^{D}<d$, then $l^{D}$ is also the smallest empty level in ORAM . For $d:=D$ to 0 , perform the following:

(a) If $d<l^{D}$, set $l:=d$; otherwise, set $l:=l^{D}$.

(b) Call $U \leftarrow \mathrm{ORAM}_{d}$.Shuffle $\left(\left(\mathrm{OTM}_{0}^{d}, \ldots, \mathrm{OTM}_{l}^{d}, U^{d}\right), l\right)$.

Recall that to complete the description of Shuffle, we need to specify the update rule that determines how to combine the values of the same address that appears in both the current ORAM $_{d}$ and $U^{d}$.

For $d<D$, in $U^{d}$ and ORAM $_{d}$, each depth- $d$ logical address addr ${ }^{\langle d\rangle}$ stores the position labels for both children addresses addr ${ }^{\langle d\rangle} \| 0$ and $\operatorname{addr}^{\langle d\rangle} \| 1$ (in depth $d+1$ ). For each of the child addresses, if $U^{d}$ contains a new position label, choose the new one; otherwise, choose the old label previously in ORAM ${ }_{d-1}$.

(c) If $d \geq 1$, we need to send the updated positions involved in $U$ to depth $d-1$. We use the Convert subroutine (detailed description below) to convert $U$ into 
an update array for depth- $(d-1)$ addresses, where each entry may pack the position labels for up to two sibling depth- $d$ addresses.

Set $U^{d-1} \leftarrow$ Convert $(U, d)$, which will be used in the next iteration for recursion depth $d-1$ to perform its shuffle.

The Convert subroutine. $U$ is a sorted, unpermuted layout representing the abstract array $\left\{\left(\operatorname{addr}_{i}^{\langle d\rangle}, \operatorname{pos}_{i}\right): i \in[|U|]\right\}$. The subroutine Convert $(U, d)$ proceeds as follows.

For $i:=0$ to $|U|$, the client reconstructs $\left(\operatorname{addr}_{i-1}^{\langle d\rangle}, \operatorname{pos}_{i-1}\right),\left(\operatorname{addr}_{i}^{\langle d\rangle}, \operatorname{pos}_{i}\right)$ and $\left(\operatorname{addr}_{i+1}^{\langle d\rangle}, \operatorname{pos}_{i+1}\right)$, computes $u_{i}^{\prime}$ using the rules below and secretly writes $u_{i}^{\prime}$ to $U^{d-1}$.

- If $\operatorname{addr}_{i}^{\langle d\rangle}=\operatorname{addr} \| 0$ and $\operatorname{addr}_{i+1}^{\langle d\rangle}=\operatorname{addr} \| 1$ for some addr, i.e., if my right neighbor is my sibling, then write down $u_{i}^{\prime}:=\left(\operatorname{addr},\left(\operatorname{pos}_{i}, \operatorname{pos}_{i+1}\right)\right)$, i.e., both siblings' positions need to be updated.

- If $\operatorname{addr}_{i-1}^{\langle d\rangle}=\operatorname{addr} \| 0$ and $\operatorname{addr}_{i}^{\langle d\rangle}=\operatorname{addr} \| 1$ for some addr, i.e., if my left neighbor is my sibling, then write down $u_{i}^{\prime}:=\perp$.

- Else if $i$ does not have a neighboring sibling, parse $\operatorname{addr}_{i}^{\langle d\rangle}=\operatorname{addr} \| b$ for some $b \in\{0,1\}$, then write down $u_{i}^{\prime}:=\left(\operatorname{addr},\left(\operatorname{pos}_{i}, *\right)\right)$ if $b=0$ or write down $u_{i}^{\prime}:=\left(\operatorname{addr},\left(*, \operatorname{pos}_{i}\right)\right)$ if $b=1$. In these cases, only the position of one of the siblings needs to be updated in ORAM $_{d-1}$.

- Let $U^{d-1}:=\left\{u_{i}^{\prime}: i \in[|U|]\right\}$. Note here that each entry of $U^{d-1}$ contains a depth- $(d-1)$ address of the form addr, as well as the update instructions for two position labels of the depth- $d$ addresses addr $\| 0$ and addr $\| 1$ respectively. We emphasize that when * appears, this means that the position of the corresponding depth- $d$ address does not need to be updated in ORAM $_{d-1}$.

- Output $U^{d-1}$.

Lemma 3. The above ORAM scheme is perfectly oblivious in the presence of a semi-honest adversary corrupting a single server.

Proof. Observe that we adopt the framework of building a recursive ORAM, where each depth is a position-based ORAM. Such a framework has been used for perfectly secure ORAM with one-server [5] assuming perfect secure cryptography.

As mentioned in Lemma 2, the major difference here is that the data is secretly shared between three servers, thereby eliminating the need of perfectly secure cryptography. In a fashion similar to [5], the security of our recursive ORAM derives its security from the underlying position-based ORAMs, because the overall view of the adversary is composed of the views corresponding to the position-based ORAMs in all the recursion depths, plus deterministic access pattern to pass data (which is secretly shared by independent randomness) between successive recursion depths. Since our position-based ORAM in each recursion depth is perfectly oblivious, so is our overall recursive ORAM.

Fact 15 Each ORAM access takes an amortized bandwidth blowup of $O\left(\log ^{2} N\right)$. 
Proof. There are $D=O(\log N)$ position-based ORAMs in the construction. For each $0 \leq d \leq D$, we analyze the number of block operations associated with $\mathrm{ORAM}_{d}$. From Fact 10 , the bandwidth required by the fetch phase is $O(d)$. From Fact 14, building the OTM at level $\ell$ takes a bandwidth of $O\left(2^{\ell}\right)$. However, since an level- $\ell$ is rebuilt every $\Theta\left(2^{\ell}\right)$ ORAM accesses, the amortized bandwidth to rebuild each level is $O(1)$. Hence, the amortized bandwidth for the maintain phase is $O(d)$. Therefore, the total amortized bandwidth per ORAM access is $\sum_{d=1}^{D} d=O\left(\log ^{2} N\right)$, which is also the bandwidth blowup.

Summarizing the above, we arrive at the following main theorem:

Theorem 16 (Perfectly secure 3-server ORAM). There exists a 3-server ORAM scheme that satisfies perfect correctness and perfect security against a semi-honest adversary in control of any single server, and achieves $O\left(\log ^{2} N\right)$ amortized bandwidth blowup (where $N$ denotes the total number of logical blocks).

Finally, similar to existing works that rely on the recursion technique 23.25, we can achieve better bandwidth blowup with larger block sizes: suppose each data block is at least $\Omega\left(\log ^{2} N\right)$ in size, and we still set the position map blocks to be $O(\log N)$ bits long, then our scheme achieves $O(\log N)$ bandwidth blowup.

\section{Acknowledgments}

T-H. Hubert Chan was supported in part by the Hong Kong RGC under grant 17200418. Jonathan Katz was supported in part by NSF award \#1563722. Kartik Nayak was supported by a Google Ph.D. fellowship. Antigoni Polychroniadou was supported by the Junior Simons Fellowship. Elaine Shi was supported in part by NSF award CNS-1601879, a Packard Fellowship, and a DARPA Safeware grant (subcontractor under IBM).

\section{References}

1. I. Abraham, C. W. Fletcher, K. Nayak, B. Pinkas, and L. Ren. Asymptotically tight bounds for composing ORAM with PIR. In PKC, 2017.

2. M. Ajtai. Oblivious RAMs without cryptographic assumptions. In STOC, 2010.

3. T.-H. H. Chan, Y. Guo, W.-K. Lin, and E. Shi. Oblivious hashing revisited, and applications to asymptotically efficient ORAM and OPRAM. In Asiacrypt, 2017.

4. T.-H. H. Chan, Y. Guo, W.-K. Lin, and E. Shi. Cache-oblivious and data-oblivious sorting and applications. In SODA, 2018.

5. T.-H. H. Chan, K. Nayak, and E. Shi. Perfectly secure oblivious parallel RAM. Cryptology ePrint Archive, Report 2018/364, 2018.

6. T.-H. H. Chan and E. Shi. Circuit OPRAM: A unifying framework for computationally and statistically secure ORAMs and OPRAMs. In TCC, 2017.

7. K.-M. Chung, Z. Liu, and R. Pass. Statistically-secure ORAM with $\tilde{O}\left(\log ^{2} n\right)$ overhead. In Asiacrypt, 2014.

8. I. Damgård, S. Meldgaard, and J. B. Nielsen. Perfectly secure oblivious RAM without random oracles. In TCC, pages 144-163, 2011. 
9. I. Demertzis, D. Papadopoulos, and C. Papamanthou. Searchable encryption with optimal locality: Achieving sublogarithmic read efficiency. Cryptology ePrint Archive, Report 2017/749, 2017. https://eprint.iacr.org/2017/749

10. K. Donald et al. The art of computer programming, volume 2: Semi numerical algorithms, 1998.

11. O. Goldreich. Towards a theory of software protection and simulation by oblivious RAMs. In $S T O C, 1987$.

12. O. Goldreich and R. Ostrovsky. Software protection and simulation on oblivious RAMs. J. ACM, 1996.

13. M. T. Goodrich. Data-oblivious external-memory algorithms for the compaction, selection, and sorting of outsourced data. In SPAA, 2011.

14. M. T. Goodrich and M. Mitzenmacher. Privacy-preserving access of outsourced data via oblivious RAM simulation. In ICALP, pages 576-587, 2011.

15. D. Gordon, J. Katz, and X. Wang. Simple and efficient two-server ORAM. In Asiacrypt, 2018.

16. T. Hoang, C. D. Ozkaptan, A. A. Yavuz, J. Guajardo, and T. Nguyen. S3ORAM: A computation-efficient and constant client bandwidth blowup ORAM with shamir secret sharing. In $C C S, 2017$.

17. E. Kushilevitz, S. Lu, and R. Ostrovsky. On the (in)security of hash-based oblivious RAM and a new balancing scheme. In SODA, 2012.

18. E. Kushilevitz and T. Mour. Sub-logarithmic distributed oblivious RAM with small block size. CoRR, abs/1802.05145, 2018.

19. W.-K. Lin, E. Shi, and T. Xie. Can we overcome the $n \log n$ barrier for oblivious sorting? Cryptology ePrint Archive, Report 2018/227, 2018.

20. S. Lu and R. Ostrovsky. Distributed oblivious RAM for secure two-party computation. In Theory of Cryptography Conference (TCC), pages 377-396, 2013.

21. M. Raskin and M. Simkin. Oblivious RAM with small storage overhead. Cryptology ePrint Archive, Report 2018/268, 2018. https ://eprint.iacr.org/2018/268.

22. L. Ren, C. W. Fletcher, A. Kwon, E. Stefanov, E. Shi, M. Van Dijk, and S. Devadas. Constants count: Practical improvements to oblivious RAM. In USENIX Security Symposium, pages 415-430, 2015.

23. E. Shi, T.-H. H. Chan, E. Stefanov, and M. Li. Oblivious RAM with $O\left((\log N)^{3}\right)$ worst-case cost. In ASIACRYPT, pages 197-214, 2011.

24. E. Stefanov and E. Shi. Multi-cloud oblivious storage. In CCS, 2013.

25. E. Stefanov, M. van Dijk, E. Shi, C. Fletcher, L. Ren, X. Yu, and S. Devadas. Path ORAM - an extremely simple oblivious RAM protocol. In CCS, 2013.

26. S. Tople, H. Dang, P. Saxena, and E.-C. Chang. Permuteram: Optimizing oblivious computation for efficiency. Cryptology ePrint Archive, Report 2017/885, 2017.

27. X. S. Wang, T.-H. H. Chan, and E. Shi. Circuit ORAM: On Tightness of the Goldreich-Ostrovsky Lower Bound. In ACM CCS, 2015. 